\title{
Undermining ribosomal RNA transcription in both the nucleolus and mitochondrion: an offbeat approach to target MYC-driven cancer
}

\author{
Stefano Rossetti ${ }^{1}$, Andrzej J. Wierzbicki ${ }^{1}$ and Nicoletta Sacchi ${ }^{1}$ \\ ${ }^{1}$ Department of Cancer Genetics and Genomics, Roswell Park Cancer Institute, Buffalo, NY 14263, USA \\ Correspondence to: Nicoletta Sacchi, email: nicoletta.sacchi@roswellpark.org \\ Keywords: Pol I-nucleolar rRNA transcription; POLRMT-mitochondrial rRNA transcription; MYC-driven proliferation; Pol I and POLRMT \\ inhibitors \\ Received: September 18, $2017 \quad$ Accepted: December 09, $2017 \quad$ Published: December 22, 2017 \\ Copyright: Rossetti et al. This is an open-access article distributed under the terms of the Creative Commons Attribution License \\ 3.0 (CC BY 3.0), which permits unrestricted use, distribution, and reproduction in any medium, provided the original author and \\ source are credited.
}

\section{ABSTRACT}

The MYC transcription factor coordinates, via different RNA polymerases, the transcription of both ribosomal RNA (rRNA) and protein genes necessary for nucleolar as well as mitochondrial ribogenesis. In this study we tested if MYC-coordination of rRNA transcription in the nucleolus and in the mitochondrion drives (cancer) cell proliferation. Here we show that the anti-proliferative effect of CX-5461, a Pol I inhibitor of rRNA transcription, in ovarian (cancer) cell contexts characterized by MYC overexpression is enhanced either by 2'-C-Methyl Adenosine (2'-C-MeA), a ribonucleoside that inhibits POLRMT mitochondrial rRNA (mt-rRNA) transcription and doxycycline, a tetracycline known to affect mitochondrial translation. Thus, hindering not only mt-rRNA transcription, but also mitoribosome function in MYCoverexpressing ovarian (cancer) cells, potentiates the antiproliferative effect of CX-5461. Targeting MYC-regulated rRNA transcription and ribogenesis in both the nucleolus and mitochondrion seems to be a novel approach worth of consideration for treating MYC-driven cancer.

\section{INTRODUCTION}

The nucleolus is the major site of ribosomal RNA (rRNA) synthesis. Moreover, the mitochondrion is another site of rRNA synthesis. The MYC transcription factor regulates genes encoding proteins necessary for both nucleolar and mitochondrial rRNA transcription. MYC enables RNA Polymerase I (Pol I)-mediated nucleolar rRNA transcription by binding directly the rDNA promoter to induce chromatin modifications that enhance Pol I accessibility and facilitate the assembly of the Pol I transcription machinery [1-4]. MYC also controls RNA Polymerase II (Pol II)-mediated transcription of "Pol I regulon" genes encoding for basal components of the Pol I transcription machinery, including RRN3, UBTF, the SL1 complex component TAF1C, and the Pol I subunits POLR1B and POLR1E [5, 6]. In this way, MYC regulates the availability of nucleolar "Pol I regulon" factors necessary to activate rDNA genes [6]. As far as mitochondrial rRNA transcription is concerned, MYC regulates, via Pol II, the transcription of both the mitochondrial RNA polymerase POLRMT [7] and the mitochondrial transcription factor TFAM [8]. Both POLRMT and TFAM are necessary for transcription of mitochondrial DNA (mtDNA) into mitochondrial $12 \mathrm{~S}$ and 16S rRNA (mt-rRNA) [9]. Moreover, MYC via Pol II controls the transcription of several proteins required for mitoribogenesis [10]. Hence the hypothesis that targeting both nucleolar and mitochondrial rRNA synthesis in MYC-overexpressing cancer can have a potential antiproliferative value.

In cancer of different histotypes MYC gene amplification or upregulation drive tumorigenesis by inducing stemness, promoting cell growth and proliferation, and hampering cell differentiation $[11,12]$. Thus, drugs capable of inhibiting nucleolar Pol I-mediated 
rRNA transcription are emerging as new tools to target MYC-overexpressing cancer $[13,14]$. One of these drugs is CX-5461. This small molecule, by selectively inhibiting Pol I-mediated rRNA transcription, can efficiently curb cancer cell proliferation both in vitro and in vivo [15-20]. Indeed, recent studies suggest that CX-5461 effectively targets MYC-driven proliferation in different cancer cell contexts, including lymphoma [16, 21], multiple myeloma [22], and prostate cancer $[23,24]$.

Inhibition of mitochondrial ribogenesis is also a promising anti-cancer approach $[25,26]$. Mitoribogenesis can be hindered by drugs targeting POLRMT-mediated mt-rRNA transcription, like 2'C-Methyl-Adenosine (2'-C-MeA), as recently reported in acute myelogenous leukemia studies [27]. Moreover, due to the conservation between bacteria and mitochondria, antibiotics inhibiting bacterial protein synthesis (e.g. doxycycline) seem capable of inhibiting cancer cell proliferation by targeting mitochondrial translation $[7,10,26]$.

In this study we provide evidence that MYCinduced rRNA transcription both in the nucleolus and mitochondrion concur to promote the proliferation of MYC-overexpressing cancer cells. Consistently, MYC overexpression sensitizes cells to the antiproliferative action of drugs targeting on one hand nucleolar Pol I-mediated rRNA transcription (e.g. CX-5461), and on the other hand, either POLRMT-mediated mitochondrial rRNA transcription (e.g. 2'-C-MeA) or mitoribosome function (e.g. doxycycline). Moreover, both 2'-C-MeA and doxycycline, when combined with CX-5461, induce a more effective antiproliferative action on MYCoverexpressing cells. Overall, targeting MYC-regulated rRNA transcription and ribogenesis in two distinct cell sites is an unconventional approach worth of consideration for treating MYC-driven cancer.

\section{RESULTS}

\section{Evidence of MYC-induced proliferation consequent to upregulation of both "Pol I regulon" factors and rRNA transcription in human HFF fibroblasts}

MYC is known to promote nucleolar rRNA transcription through direct activation of the Pol I transcription machinery [2-4] as well as activation of Pol II-mediated transcription of MYC-target genes encoding "Pol I regulon" factors, including RRN3, UBTF, TAF1C, and the Pol I subunits POLR1B and POLR1E $[5,6]$. To start to assess the proliferation effects of MYC overexpression due to both Pol II-mediated transcription of "Pol I regulon" factors and Pol I-mediated rRNA transcription, we used a well-characterized model of MYC-overexpressing human fibroblasts (HFF-MYC) [3, 28, 29].
HFF-MYC cells (Figure 1A) express higher levels of RRN3, UBF, TAF1C, POLR1B and POLR1E mRNAs relative to control HFF (HFF-Ctrl) (Figure 1B). Consistently, HFF-MYC also displayed upregulation of rRNA transcription, which was assessed by using two different strategies: 1) qRT-PCR of the 47S pre-rRNA 5'ETS, which is spliced out from mature rRNA (Figure 1C), and 2) quantitative analysis of 5-Ethynyl Uridine (EU) incorporation into newly synthesized RNA within the B23-positive nucleolar compartment (Figure 1D).

MYC overexpression significantly promoted HFFMYC cell proliferation, which was evaluated both by both MTT assay (Figure 1E, left) and incorporation of 5-ethynyl-2'-deoxyuridine (EdU) into nascent DNA of cells in $\mathrm{S}$ phase (Figure 1E, middle and right). To further assess if upregulation of rRNA transcription contributes to MYC-induced cell proliferation, we treated cells with the Pol I inhibitor CX-5461, which prevents the formation of the Pol I pre-initiation complex [17]. Treatment with CX$5461(100 \mathrm{nM})$ significantly counteracted MYC-induced rRNA transcription (assessed as pre-rRNA) in HFF-MYC cells (Figure 1F, red bars). CX-5461 also counteracted HFFMYC proliferation in a dose-dependent manner (Figure $1 \mathrm{G}$, left, red bars). To a lesser extent, CX-5461 inhibited rRNA transcription (Figure 1F, blue bars) and, consequently, proliferation (Figure $1 \mathrm{G}$, left, blue bars) also in the HFF-Ctrl normal cell context. However, when HFF-MYC and HFF$\mathrm{Ctrl}$ proliferation data were normalized to their respective untreated controls, HFF-MYC showed significantly higher sensitivity to the anti-proliferative action of CX-5461 relative to normal HFF-Ctrl (Figure $1 \mathrm{G}$, right).

Overall, these preliminary results in a human fibroblast cell context indicate that MYC-induced proliferation can be traced to increased rRNA transcription due to: a) MYC-induced direct activation of Pol I, and b) MYC-induced upregulation of "Pol I regulon" factors. Moreover, MYC overexpression sensitizes HFF cells to the anti-proliferative action of the Pol I inhibitor CX-5461.

\section{Increased proliferation consequent to upregulation of both "Pol I regulon" genes and rRNA transcription in MYC-overexpressing human ovarian epithelial cells}

According to The Cancer Genome Atlas, MYC amplification/upregulation is frequent in cancer, including ovarian cancer, where it is detected in over $30 \%$ of cases [30]. For this reason, we tested if MYC overexpression in ovarian cancer induces Pol I-mediated rRNA transcription as well as Pol II-mediated transcription of "Pol I regulon" genes. As shown here, CAOV4 ovarian cancer cells, which overexpress MYC (Figure 2A), display increased levels of rRNA transcription (assessed as pre-rRNA) (Figure 2B) and upregulation of "Pol I regulon" genes (Figure 2C) relative to $\mathrm{CAOV} 3$ ovarian cancer cells, which do not overexpress MYC. 
Next, we set out to test the effects of different levels of MYC overexpression on nucleolar rRNA transcription in IOSE and HOSE human ovarian epithelial cells. Using telomerase-immortalized IOSE cells [31] we developed cells with increasing expression levels of exogenous MYC: IOSE-MYC, with MYC expression higher than control IOSE-Ctrl cells, and IOSE-MYC2, with a MYC overexpression level higher than IOSE-MYC (Figure 3A). The expression of "Pol I regulon" target genes (RRN3, UBF, TAF1C, POLR1B, POLR1E) was significantly higher in MYC-overexpressing IOSE lines than in IOSE-Ctrl, and correlated with MYC level (Figure 3B).

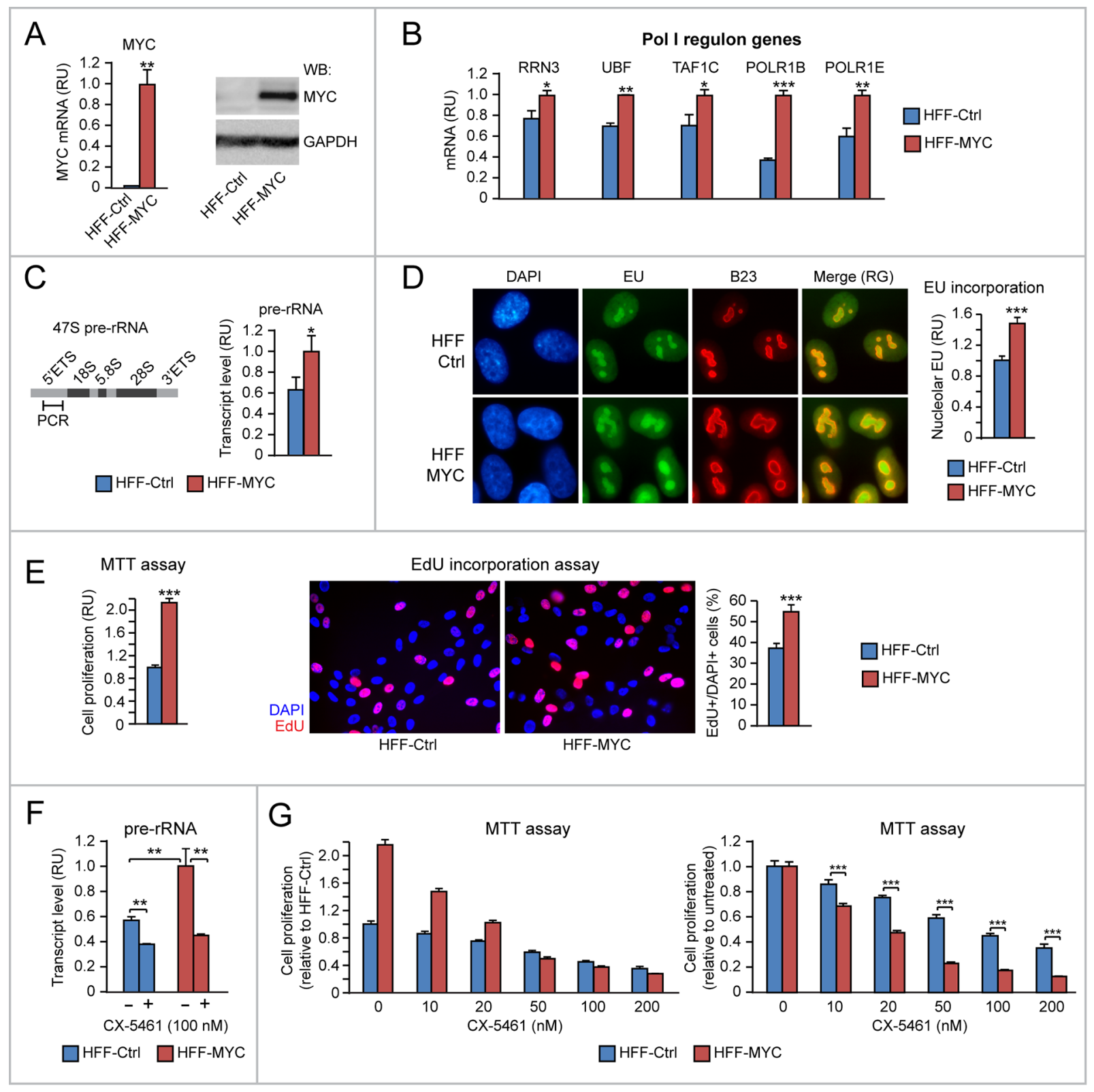

Figure 1: Evidence of MYC-induced proliferation consequent to upregulation of both "Pol I regulon" factors and rRNA transcription in human HFF fibroblasts. (A-B) HFF-MYC fibroblasts overexpressing exogenous MYC (see qRT-PCR in A, left, and Western Blot in A, right) express significantly higher levels of "Pol I regulon" genes (assessed by q-RT-PCR) (B) relative to HFF-Ctrl fibroblasts. (C-D) HFF-MYC display increased nucleolar rRNA transcription relative to HFF-Ctrl, as measured by qRT-PCR of pre-rRNA 5'ETS (C) as well as by immunofluorescent quantification of EU incorporation in the B23-positive nucleolar compartment (D). Nucleolar EU incorporation was digitally quantified with Photoshop (see Materials and Methods for details). (E) MTT assay (left) and EdU incorporation analysis (middle and right) show that HFF-MYC cells proliferate significantly more than HFF-Ctrl cells. (F-G) Treatment with the Pol I inhibitor CX-5461, by reducing nucleolar rRNA transcription (F), inhibits proliferation of HFF-MYC cells significantly more than HFF-Ctrl cells (G). The chart in G, left, shows values normalized to untreated HFF-Ctrl; the chart in G, right, shows values normalized to each untreated cell line. ${ }^{*} \mathrm{p}<0.05,{ }^{* *} \mathrm{p}<0.01,{ }^{* * *} \mathrm{p}<0.001$. 
Moreover, MYC-overexpressing IOSE displayed increased rRNA transcription, which was assessed both by pre-rRNA qRT-PCR (Figure 3C, left) and nucleolar EU incorporation (Figure 3C, middle and right). Consistently, we detected a MYC level-dependent increase of cell proliferation in IOSE-MYC and IOSE-MYC2 relative to IOSE-Ctrl (see MTT assay in Figure 3D, left, and EdU incorporation assay in Figure 3D, middle and right). We obtained similar findings also in SV40-immortalized HOSE cells (Supplementary Figure 1A-1D).

Thus, regardless of the cell context, increasing MYC overexpression seems sufficient to differentially foster cell proliferation by inducing Pol II-dependent transcription of "Pol I regulon" genes as well as Pol I-dependent rRNA synthesis.

\section{Additional evidence that upregulation of "Pol I regulon" genes contributes to promote cell proliferation in MYC-overexpressing IOSE cells}

To mechanistically prove that increased proliferation of MYC-overexpressing IOSE cells was due, in part, to upregulation of "Pol I regulon" genes and rRNA transcription, we transiently transfected IOSE-MYC2 cells with short interfering RNAs (siRNAs) targeting either MYC, or either one of two key components of the "Pol I regulon": RRN3 and UBF.

As shown in Figure 4A, transient MYC knock down by siMYC in IOSE-MYC2 cells (left) resulted in downregulation of both RRN3 and UBF mRNAs, as well as in decreased pre-rRNA level (right) relative to cells transfected with control siRNA (siCtrl). Importantly, MYC knock down also counteracted MYC-induced proliferation of IOSE-MYC2 cells (see EdU incorporation assay in Figure 4B). In turn, transient knock down of either RRN3 (Figure 4C, left) or UBF (Figure 4C, right) in IOSEMYC2 was sufficient to significantly reduce both rRNA transcription (see pre-rRNA qRT-PCR in Figure 4C, left and right), and cell proliferation (see EdU incorporation assay in Figure 4D).

Based on these findings, the pro-proliferative effects of MYC overexpression in human ovarian epithelial cells rely, at least in part, on MYC transcriptional ability to upregulate "Pol I regulon" genes.

\section{MYC overexpression sensitizes human ovarian epithelial cells to the anti-proliferative action of the Pol I inhibitor CX-5461}

Selective Pol I inhibitors have been rapidly emerging as drugs capable of targeting increased rRNA synthesis $[19,32]$. Based on preliminary evidence of an antiproliferative effect of the Pol I inhibitor CX-5461 in MYC-overexpressing HFF (see Figure 1G), we did test if MYC-induced rRNA transcription was also capable of sensitizing IOSE cells to the anti-proliferative action of CX-5461.

As shown in Figure 5A, CX-5461 $(100 \mathrm{nM})$ reduced rRNA transcription (assessed by pre-rRNA quantitative RT-PCR) significantly more in IOSE-MYC2 relative to

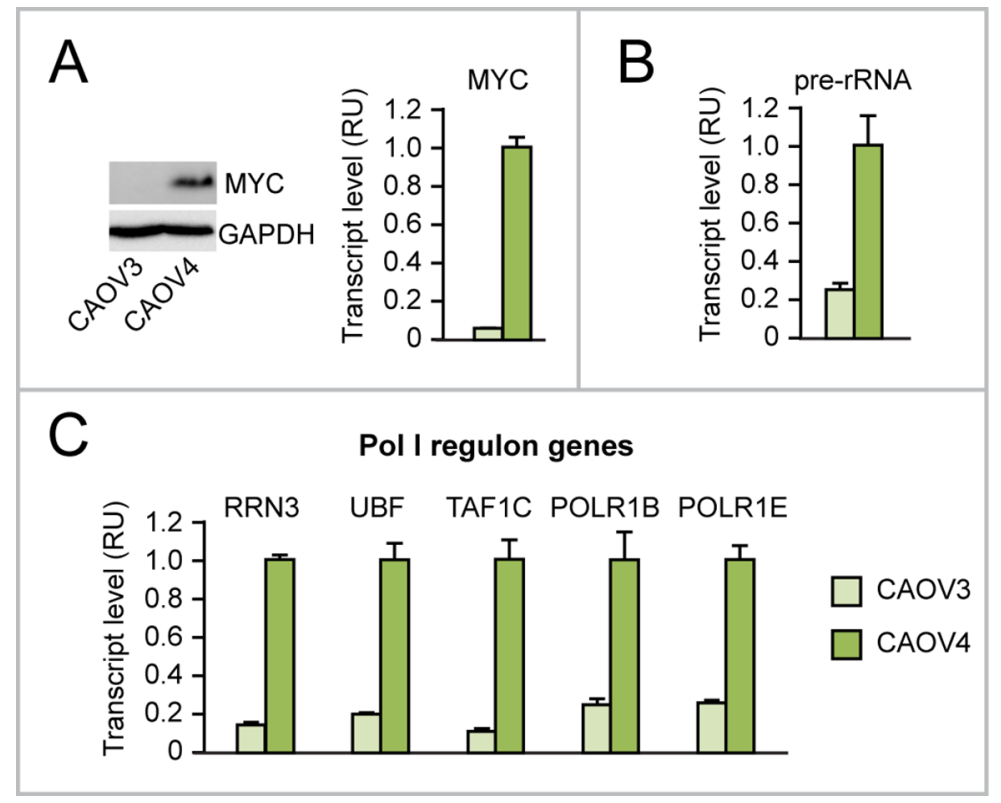

Figure 2: Evidence that MYC overexpression in ovarian cancer cells is associated with upregulation of rRNA and "Pol I regulon" factors. (A-C) Endogenous MYC overexpression in CAOV4 ovarian cancer cells (see Western Blot in A, left, and qRT-PCR in A, right) is associated with increased rRNA transcription (assessed by pre-rRNA qRT-PCR) (B) and increased expression of "Pol I regulon" genes (assessed by qRT-PCR) (C) relative to CAOV3 ovarian cancer cells, with no MYC overexpression. All differences between CAOV4 and CAOV3 are significant $(\mathrm{p}<0.05)$. 
IOSE-Ctrl. Consistently, IOSE-MYC2 cells also displayed increased sensitivity to the anti-proliferative action of CX5461 even at the lowest concentration (Figure 5B). Indeed by EdU incorporation we found that at $10 \mathrm{nM}, \mathrm{CX}-5461$ reduced the number of cells in $\mathrm{S}$ phase significantly more in IOSE-MYC2 than in IOSE-Ctrl cells (Figure 5C). A similar antiproliferative response to CX-5461 was also found in HOSE cells (Supplementary Figure 1E).

Overall, these findings indicated that a) upregulation of Pol I-mediated rRNA transcription contributes to MYCinduced proliferation in ovarian epithelial cells, and b) MYC-overexpressing human ovarian epithelial cells are more sensitive to the anti-proliferative action of the Pol I inhibitor CX-5461.

\section{Upregulation of genes involved in both mitochondrial rRNA transcription and mitoribogenesis contributes to MYC- induced HFF proliferation}

Recent studies in different cell contexts indicate that MYC can promote mitochondrial ribogenesis by activating Pol II-dependent transcription of the mitochondrial RNA polymerase POLRMT and the mitochondrial transcription factor TFAM [7, 8]. Further, MYC can induce Pol IIdependent transcription of several mitoribosome proteins (e.g. PTCD3, MRPS5, and MRPS27) [10].

We used the HFF model to assess whether the effects of MYC overexpression on proliferation can

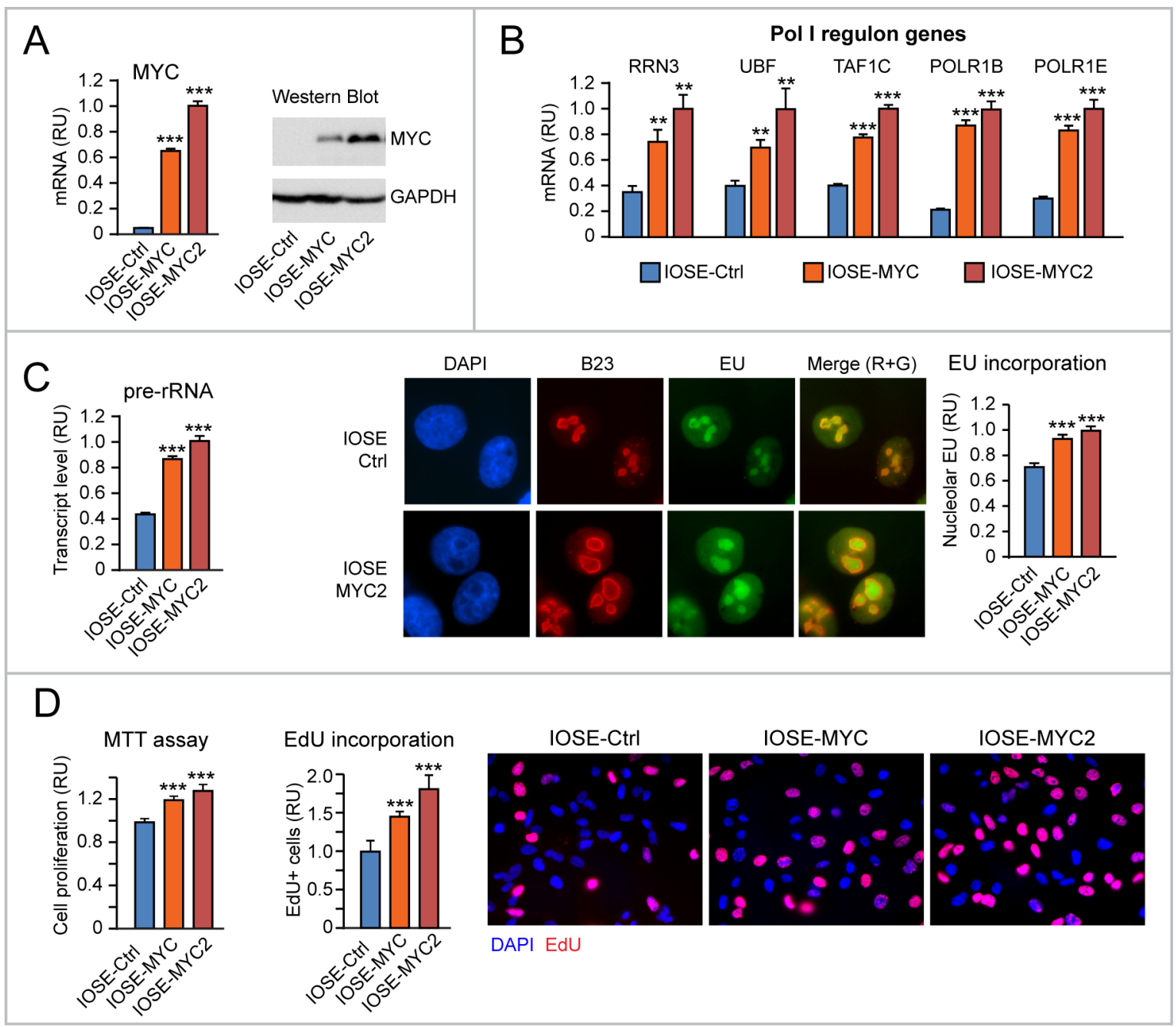

Figure 3: Increased proliferation consequent to upregulation of both "Pol I regulon" genes and rRNA transcription in MYC-overexpressing human ovarian epithelial cells. (A-B) Increasing expression of exogenous MYC in IOSE ovarian epithelial cells (see qRT-PCR in A, left, and Western Blot in A, right) leads to a MYC dose-dependent upregulation of "Pol I regulon" genes (assessed by qRT-PCR) relative to control IOSE cells (B). (C) Exogenous MYC expression in IOSE cells leads to upregulation of rRNA transcription, assessed both by pre-rRNA qRT-PCR (left) and quantification of EU incorporation into the B23-positive nucleolar compartment (middle and left). Nucleolar EU incorporation was digitally quantified with Photoshop (see Materials and Methods for details). (D) Exogenous MYC expression promotes IOSE proliferation, as shown by both MTT assay (left) and EdU incorporation (middle and right). ${ }^{* *} \mathrm{p}<0.01$, ${ }^{* * *} \mathrm{p}<0.001$. 


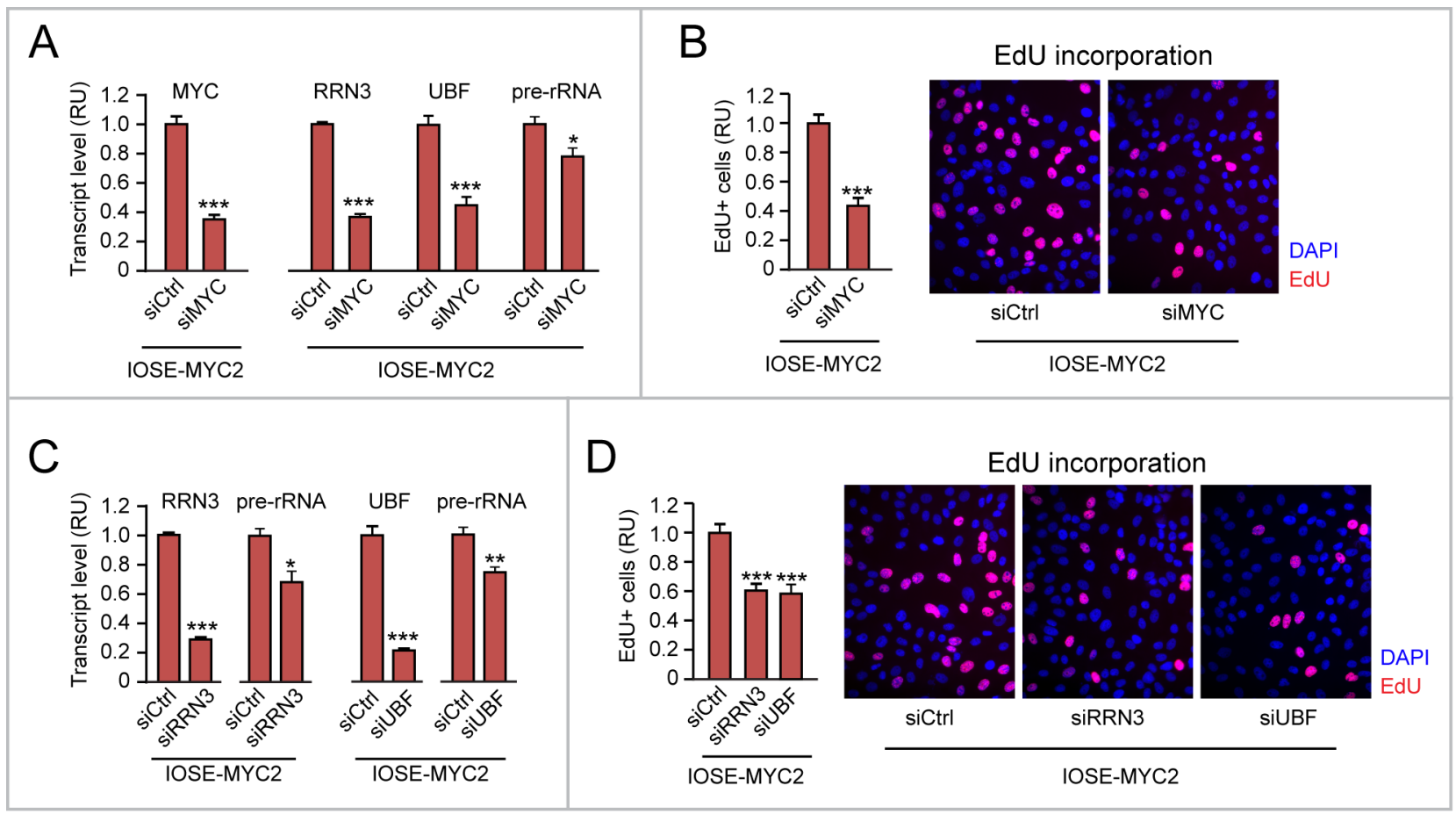

Figure 4: Additional evidence that upregulation of "Pol I regulon" genes contributes to promote cell proliferation in MYC-overexpressing IOSE cells. (A-B) Decreasing the level of exogenous MYC in IOSE-MYC2 cells by transient siRNA (see qRT-PCR in A, left) is sufficient to significantly decrease the transcript levels of RRN3, UBF, and pre-rRNA (see qRT-PCR in A, right) as well as cell proliferation (see EdU incorporation in B). (C-D) Transient knock down of either RRN3 or UBF in IOSE-MYC2 significantly decreases pre-rRNA level (see qRT-PCR in C) and cell proliferation (see EdU incorporation in D). ${ }^{*} \mathrm{p}<0.05,{ }^{* *} \mathrm{p}<0.01,{ }^{* * *} \mathrm{p}<0.001$.

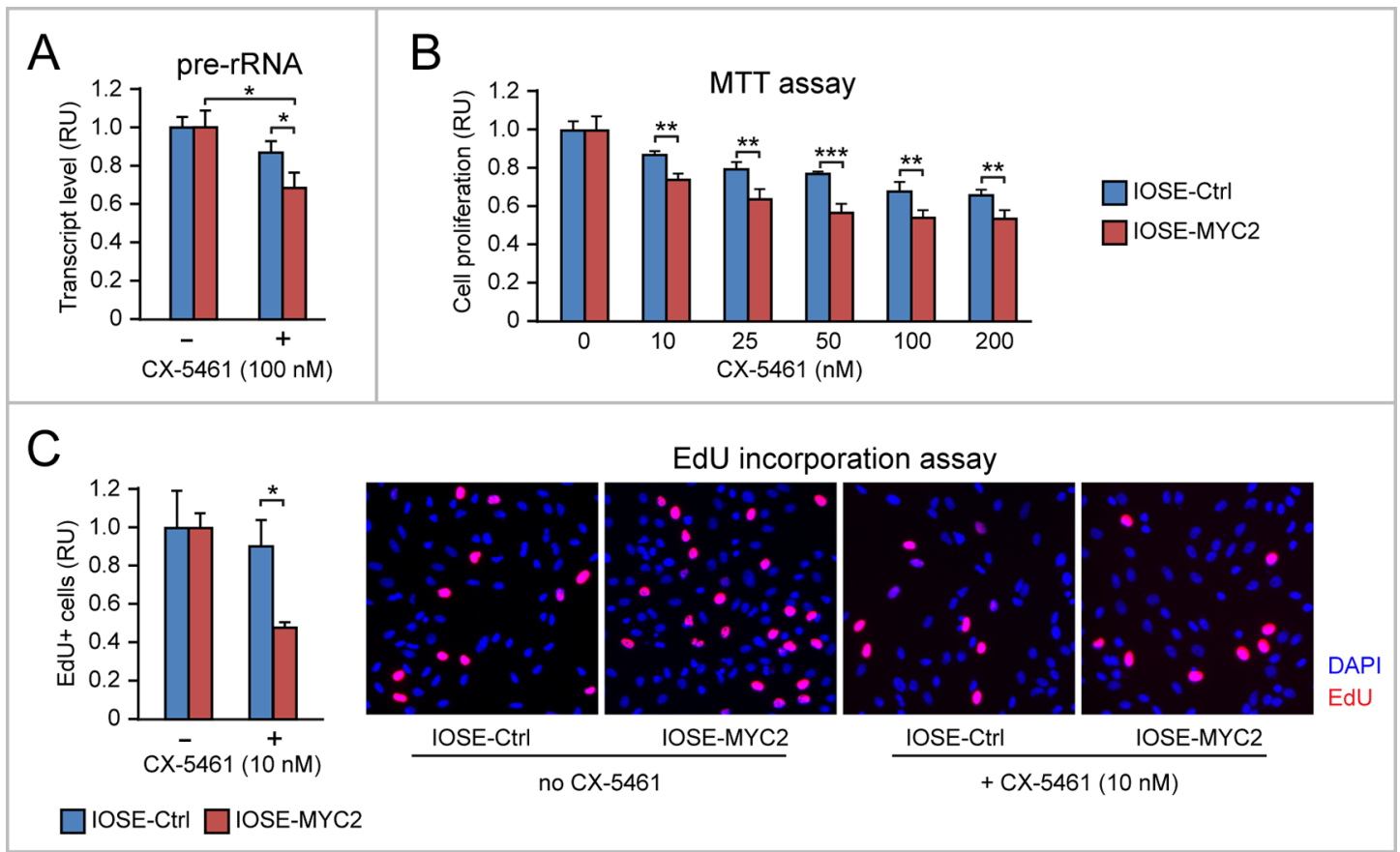

Figure 5: MYC overexpression sensitizes human ovarian epithelial cells to the anti-proliferative action of the Pol I inhibitor CX-5461. (A) The Pol I inhibitor CX-5461 reduces rRNA transcription (assessed by pre-rRNA qRT-PCR) significantly more in IOSE-MYC2 than in IOSE-Ctrl cells. (B-C) Consistently, IOSE-MYC2 are more sensitive than IOSE-Ctrl cells to the anti-proliferative action of CX-5461, as shown by both MTT assay (B) and analysis of EdU incorporation by immunofluorescence $(\mathrm{C}) .{ }^{*} \mathrm{p}<0.05,{ }^{* *} \mathrm{p}<0.01$, ${ }^{* * *} \mathrm{p}<0.001$. 
be traced to either increased mt-rRNA transcription or upregulation of mitoribosome proteins. Relative to HFFCtrl, HFF-MYC cells displayed increased levels of both POLRMT and TFAM mRNAs (Figure 6A, left), as well as increased levels of three pre-mtRNA regions (Figure 6A, middle and right): i) a region within the $12 \mathrm{~S}$ mitochondrial RNA, ii) a region within the $16 \mathrm{~S} \mathrm{mtRNA}$, and iii) a region across the $12 \mathrm{~S}$ and $16 \mathrm{~S}$ mt-rRNA. Since the $12 \mathrm{~S}$ and 16S RNAs are subsequently spliced out to form mature mitochondrial rRNAs, this last region is present only in mt-rRNA precursors, thus indirectly reflecting the rate of mt-rRNA transcription. In addition, HFF-MYC also displayed increased transcript levels of PTCD3, MRPS5, and MRPS27 mitoribosome proteins relative to HFFCtrl (Figure 6B), thus confirming that, also in this cell context, MYC overexpression can promote mitoribosome formation by concomitantly enhancing both mt-rRNA transcription and expression of mitochondrial ribosomal proteins.

To test the contribution of MYC-induced mt-rRNA transcription and mitoribogenesis to $\mathrm{MYC}$-induced proliferation, first we treated both $\mathrm{HFF}-\mathrm{Ctrl}$ and $\mathrm{HFF}$ MYC cells with 2'-C-methyladenosine (2'-C-MeA), which inhibits POLRMT-mediated transcription of mt-rRNA [33]. As shown in Figure 6C, treatment with 2'-C-MeA $(20 \mu \mathrm{M})$ significantly inhibited mt-rRNA transcription (assessed by qRT-PCR of the 12-16S region of the premtRNA) in HFF-MYC. Consistently, 2'-C-MeA also inhibited HFF-MYC proliferation in a dose-dependent fashion (see in Figure 6D, red bars). Moreover, when compared to HFF-Ctrl, HFF-MYC clearly displayed increased sensitivity to the anti-proliferative action of 2'-C-MeA (Figure 6D).

Second, we treated both HFF-Ctrl and HFF-MYC cells with doxycycline, a tetracycline that targets the $28 \mathrm{~S}$ subunit of mitochondrial ribosomes, which is homologous to the $30 \mathrm{~S}$ subunit of bacterial ribosomes. Doxycycline inhibited HFF-MYC proliferation in a dose-dependent fashion, and its anti-proliferative effects were significantly greater in the HFF-MYC cell context than in the HFF-Ctrl cell context (Figure 6E).

These preliminary findings in the HFF model showed that: i) MYC-induced mt-rRNA transcription and mitoribogenesis contribute to promote HFF-MYC proliferation; ii) MYC overexpression sensitizes HFFMYC cells to the anti-proliferative action of drugs inhibiting either mt-rRNA transcription (2'-C-MeA) or mitoribosome function (doxycycline).

\section{MYC-induced upregulation of either mt-rRNA transcription or mitoribogenesis in human ovarian epithelial cell contexts contributes to promote proliferation}

We found evidence that, relative to $\mathrm{CAOV} 3$ ovarian cancer cells, which do not overexpress MYC, CAOV4

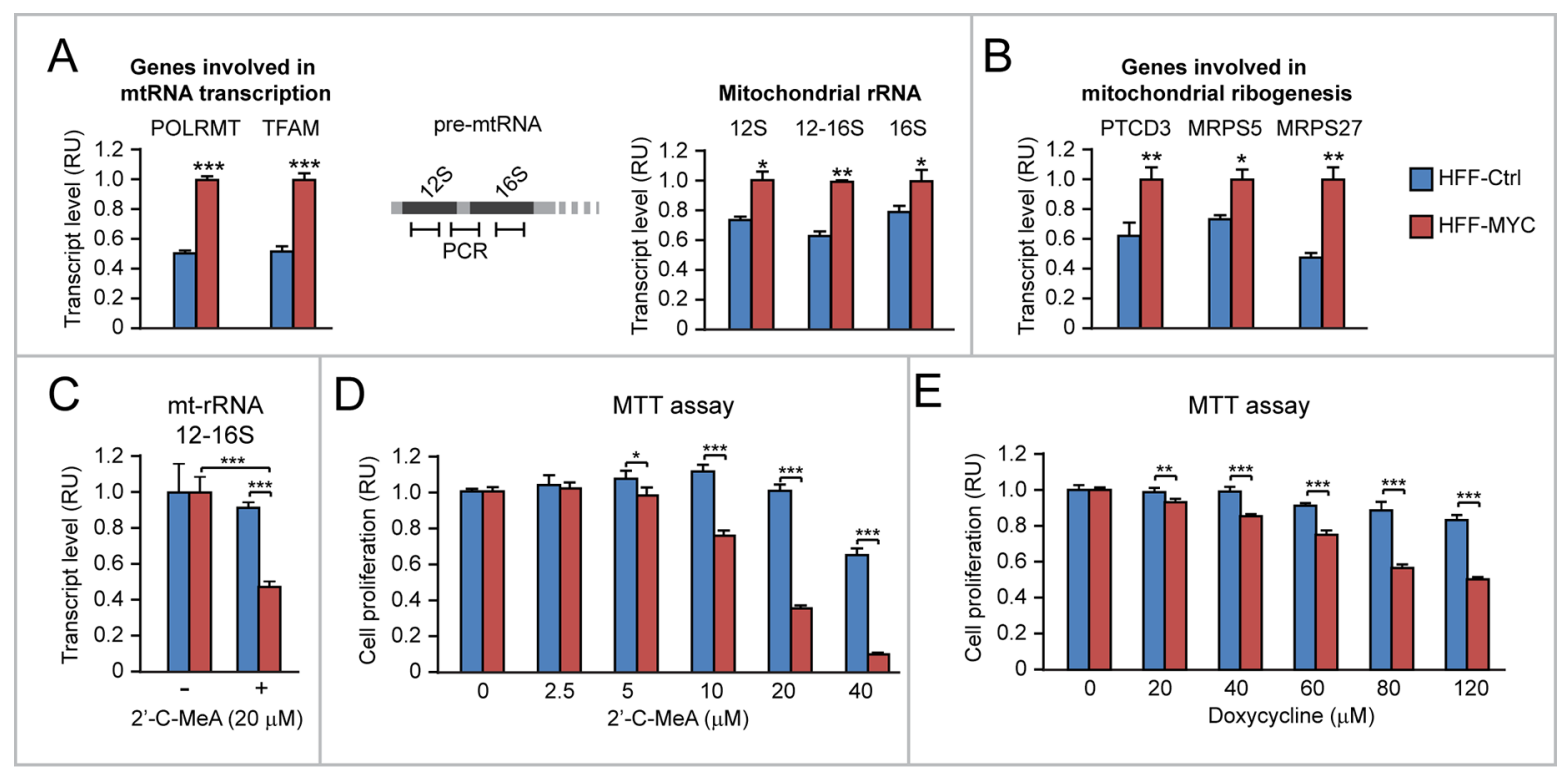

Figure 6: Upregulation of mitochondrial rRNA transcription and mitoribosome proteins contributes to MYC-induced HFF proliferation. (A) HFF-MYC, overexpressing exogenous MYC, display upregulation of genes involved in mitochondrial RNA transcription (left), as well as increased mitochondrial rRNA levels (right), which were quantified by qRT-PCR of the 12S, 16S, or 12-16S regions of pre-mtRNA (see scheme in the middle). (B) HFF-MYC cells also display upregulation of genes encoding the mitochondrial ribosomal proteins PTCD3, MRPS5, and MRPS27. (C-D) The POLRMT inhibitor 2'-C-MeA inhibits mitochondrial rRNA transcription (assessed by qRT-PCR of the 12-16S mt-rRNA) (C) and cell proliferation (assessed by MTT assay) (D) significantly more in HFF-MYC relative to HFF-Ctrl. (E) HFF-MYC are more sensitive than HFF-Ctrl to the antiproliferative action of doxycycline, which inhibits mitoribosome function. ${ }^{*} \mathrm{p}<0.05,{ }^{* *} \mathrm{p}<0.01,{ }^{* * *} \mathrm{p}<0.001$. 
ovarian cancer cells, which overexpress MYC (see Figure 2A), display a) upregulation of both MYC-target genes involved in mt-rRNA transcription (POLRMT and TFAM) and MYC-target genes encoding mitoribosome proteins (PTCD3, MRPS5, MRPS27) (Figure 7A) as well as b) increased mitochondrial rRNA synthesis (Figure 7B). For this reason, we set out to test whether MYC ectopic overexpression in human ovarian epithelial cell contexts (IOSE and HOSE) leads to increased mt-rRNA synthesis and affects mitoribogenesis.

Using the panel of IOSE cells, we detected MYC level-dependent upregulation of POLRMT, TFAM, PTCD3, MRPS5, and MRPS27 mRNAs (Figure 8A, left), as well as increased 12-16S mt-rRNA level (Figure 8A, right) in IOSE-MYC and IOSE-MYC2 cells relative to IOSE-Ctrl cells. In addition, transient MYC knock down in IOSE-MYC2 significantly reduced both POLRMT and 12-16S mt-rRNA transcript levels (Figure 8B). In turn, POLRMT knock down in IOSE-MYC2 decreased both mt-rRNA transcription (Figure 8C, left) and cell proliferation (assessed by EdU incorporation, Figure 8C, middle and right). Similar findings were obtained in the HOSE cell context (Supplementary Figure 2A-2B).

Apparently, MYC ectopic overexpression in different human ovarian epithelial cell contexts promotes mt-rRNA transcription and mitoribogenesis by upregulating MYC-target genes that are involved in these two processes. Moreover, MYC-induced mt-rRNA transcription and mitoribogenesis contribute to MYCinduced cell proliferation.

\section{Antiproliferative effect of 2'-C-MeA and doxycycline, alone or in combination with CX-5461, on MYC overexpressing ovarian epithelial cells}

Recently, it was reported that selective inhibition of either mt-rRNA transcription or mitoribosome function exerts an antiproliferative effect on MYC-overexpressing cancer cells $[7,10]$.

First, we found by MTT assay that IOSE-MYC2, relative to IOSE-Ctrl cells (Figure 9A), display increased sensitivity to the antiproliferative action of the POLRMT inhibitor 2'-C-MeA. Similar findings were also obtained in HOSE-MYC relative to HOSE-Ctrl (Supplementary Figure 2C). The 2'-C-MeA concentration of $40 \mu \mathrm{M}$, which determined the most significant difference between IOSEMYC2 and IOSE-Ctrl proliferation in the MTT assay, also decreased 12-16S mt-rRNA transcription (Figure 9B) and the number of cells in S phase (assessed by EdU incorporation) (Figure 9C) significantly more in IOSEMYC2 versus IOSE-Ctrl proliferation. Moreover, when we tested the effect of $40 \mu \mathrm{M} 2$ '-C-MeA in combination with CX5461 (ranging from $5 \mathrm{nM}$ to $100 \mathrm{nM}$ ) in IOSEMYC2 cells, we found that 2'-C-MeA significantly enhanced the antiproliferative effect of CX-5461 low concentrations (5-10 $\mathrm{nM}$ ) (Figure 9D).

Second, by MTT assay we found that IOSE-MYC2 versus IOSE-Ctrl (Figure 10A, left), as well as HOSEMYC versus HOSE-Ctrl (Supplementary Figure 2D), displayed increased sensitivity to the anti-proliferative action of doxycycline across a range of concentrations ( 0 $25 \mu \mathrm{M})$. The doxycycline concentration of $15 \mu \mathrm{M}$ showed the most significant difference between IOSE-MYC2 and IOSE-Ctrl proliferation both in the MTT assay (Figure $10 \mathrm{~A}$, left) and in the EdU incorporation assay (Figure 10A, right). Remarkably, when we tested the effect of $15 \mu \mathrm{M}$ doxycycline in combination with CX5461 (ranging from $5 \mathrm{nM}$ to $100 \mathrm{nM}$ ) on IOSE-MYC2 cells, we found that doxycycline significantly enhanced the antiproliferative effect of CX-5461 at concentrations between 5 and $25 \mathrm{nM}$ (Figure 10B).

Apparently, MYC-overexpression sensitized ovarian epithelial cells to both 2'-C-MeA and doxycycline, which target, respectively, mt-rRNA transcription and mitoribosome function. Moreover both 2'-C-MeA and

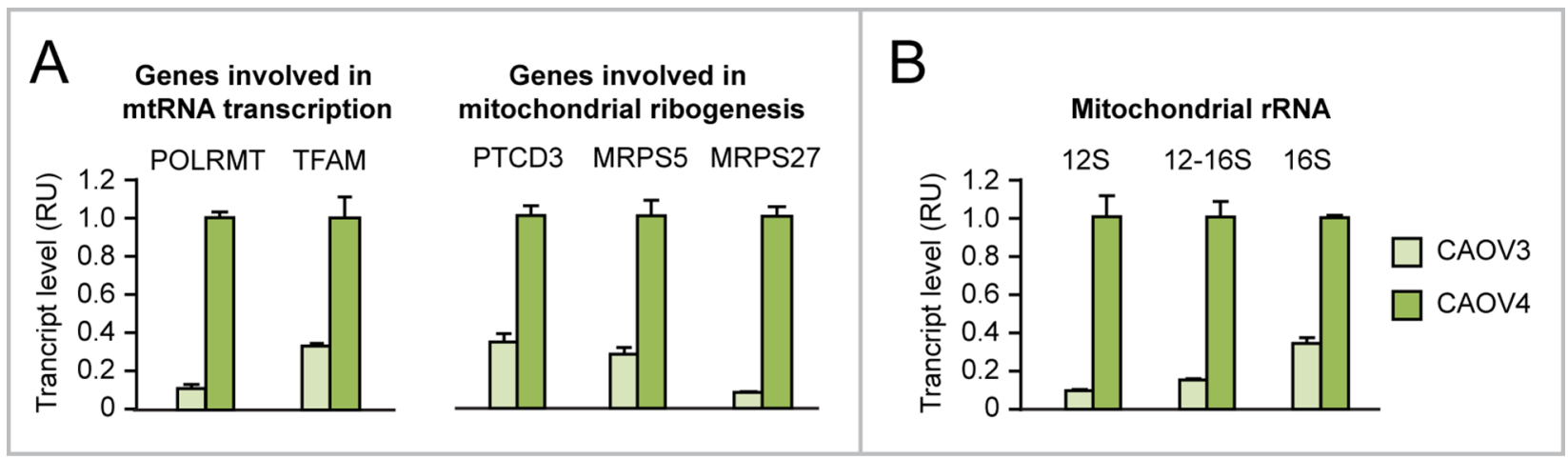

Figure 7: Evidence that MYC overexpression in ovarian cancer cells is associated with upregulation of genes involved in mitochondrial rRNA transcription and ribogenesis. (A-B) qRT-PCR showing that CAOV4 ovarian cancer cells, which overexpress endogenous MYC, display upregulation of genes involved in mitochondrial rRNA transcription (A, left) and ribogenesis (A, right) as well as increased levels of $12 \mathrm{~S}, 16 \mathrm{~S}$, and $12-16 \mathrm{~S}$ mitochondrial rRNA (B) relative to CAOV3 cells, which do not overexpress MYC. All differences between CAOV4 and CAOV3 are significant $(\mathrm{p}<0.05)$. 


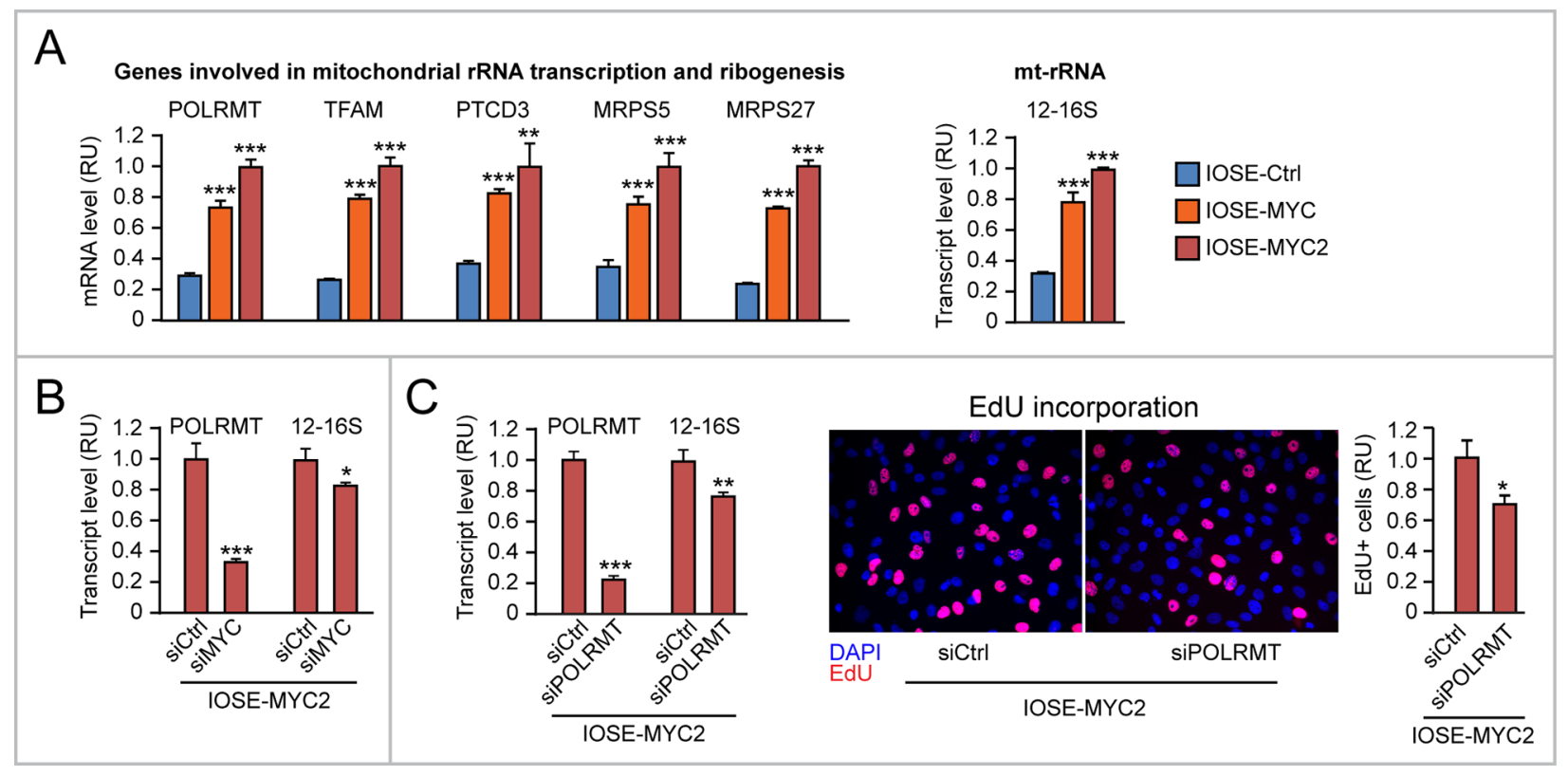

Figure 8: MYC-induced upregulation of mitochondrial rRNA transcription and ribogenesis contributes to promote proliferation of human ovarian epithelial cells. (A) qRT-PCR showing MYC dose-dependent upregulation of genes involved in mitochondrial rRNA transcription and ribogenesis (left) as well as 12-16S mt-rRNA (right) in IOSE-MYC and IOSE-MYC2 cells relative to IOSE-Ctrl. (B) Transient MYC knock down by siRNA significantly reduces both POLRMT and mt-rRNA transcription (assessed by 1216S mt-rRNA qRT-PCR) in IOSE-MYC2. (C) Transient POLRMT knock down in IOSE-MYC2 cells, by reducing mt-rRNA transcription (left), leads to decreased proliferation (assessed by EdU incorporation) (middle and right). ${ }^{*} \mathrm{p}<0.05,{ }^{* *} \mathrm{p}<0.01,{ }^{* * *} \mathrm{p}<0.001$.

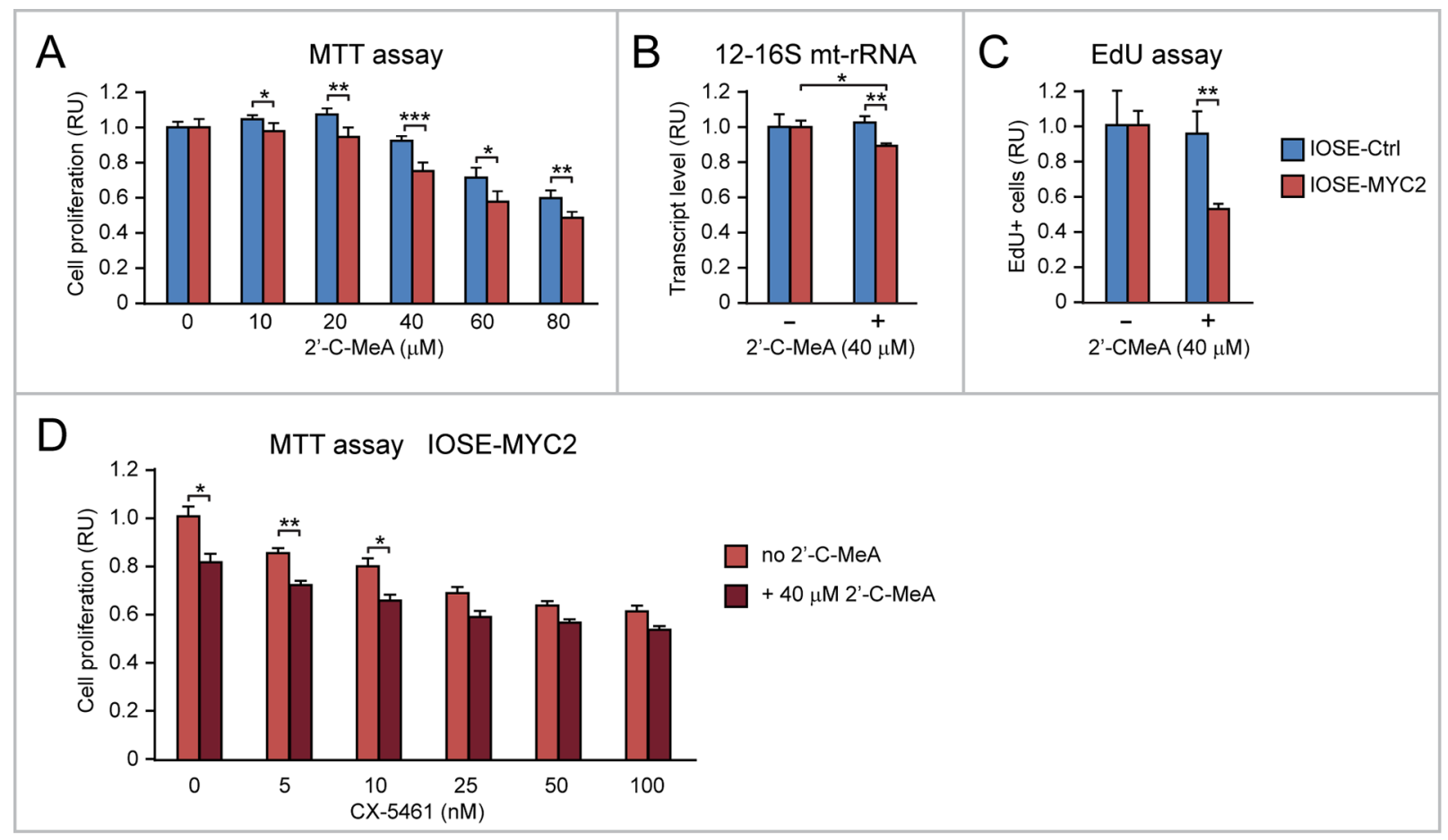

Figure 9: MYC overexpression sensitizes human ovarian epithelial cells to the anti-proliferative action of 2'-C-MeA, alone or in combination with CX-5461. (A) 2'-C-MeA inhibits cell proliferation (assessed by MTT assay) significantly more in IOSE-MYC2 that IOSE-Ctrl. (B-C) 2'-C-MeA $(40 \mu \mathrm{M})$ reduces both mt-rRNA transcription (assessed by qRT-PCR of 12-16S mt-rRNA) (B) and the number of cells in S phase (assessed by EdU incorporation) (C) significantly more in IOSE-MYC2 than in IOSE-Ctrl cells. (D) MTT assay showing that 2'-C-MeA $(40 \mu \mathrm{M})$ significantly enhances the antiproliferative effect of CX-5461 (5-10 nM) in IOSE-MYC2 cells. ${ }^{*} \mathrm{p}<0.05,{ }^{* *} \mathrm{p}<0.01,{ }^{* * *} \mathrm{p}<0.001$. 
doxycycline potentiated the antiproliferative effect of low doses of the CX-5461 inhibitor of Pol I-mediated nucleolar rRNA transcription.

\section{Both 2'-C-MeA and doxycycline can enhance the antiproliferative effects of CX-5461 in MYC- overexpressing ovarian cancer cells}

In this study we found that CAOV4 ovarian cancer cells overexpressing endogenous MYC show upregulation of nucleolar rRNA and "Pol I regulon" genes (Figure 2) as well as increased mitochondrial rRNA transcription and upregulation of MYC-target genes involved in mitochondrial rRNA transcription and ribogenesis (Figure 7). Dose-dependent inhibition of Pol I-mediated rRNA transcription with CX-5461 (Figure 11A, left) significantly reduced CAOV4 cell proliferation (Figure 11A, right). Treatment with either $2^{\prime} \mathrm{C}-\mathrm{MeA}$ (Figure 11B, left) or doxycycline (Figure 11B, right) also led to dose-dependent inhibition of CAOV4 cell proliferation. Moreover, the anti-proliferative effects of CX-5461 on CAOV4 was significantly enhanced by co-treatment with either $2^{\prime} \mathrm{C}-\mathrm{MeA}(80$ or $160 \mu \mathrm{M}$, Figure $11 \mathrm{C})$ or doxycycline (15 or $25 \mu \mathrm{M}$, Figure 11D), which were among the lowest concentrations able to induce significant inhibition of CAOV4 proliferation. In conclusion, combining CX-5461 with drugs inhibiting either mt-rRNA transcription (e.g. $2^{\prime} \mathrm{C}-\mathrm{MeA}$ ) or mitoribosome function (e.g. doxycycline) seems to be an effective antiproliferative strategy to treat MYC-overexpressing ovarian cancer.

\section{DISCUSSION}

Increasing knowledge of MYC-coordinated regulation of functions involved in both nucleolar and mitochondrial rRNA transcription and ribogenesis can open new avenues to treat MYC-driven cancer. In this study, we provide evidence of the added value of pharmacological targeting mitochondrial POLRMTmediated mt-rRNA transcription or mitoribosome translational function in conjunction with a wellestablished inhibitor of nucleolar Pol I-mediated rRNA transcription in MYC-overexpressing (cancer) cells (Figure 12).

Evidence that nucleolar rRNA transcription and ribogenesis are frequently upregulated in cancer due to different etiological factors has led to the development of novel small molecules capable of inhibiting Pol I [15, 19,

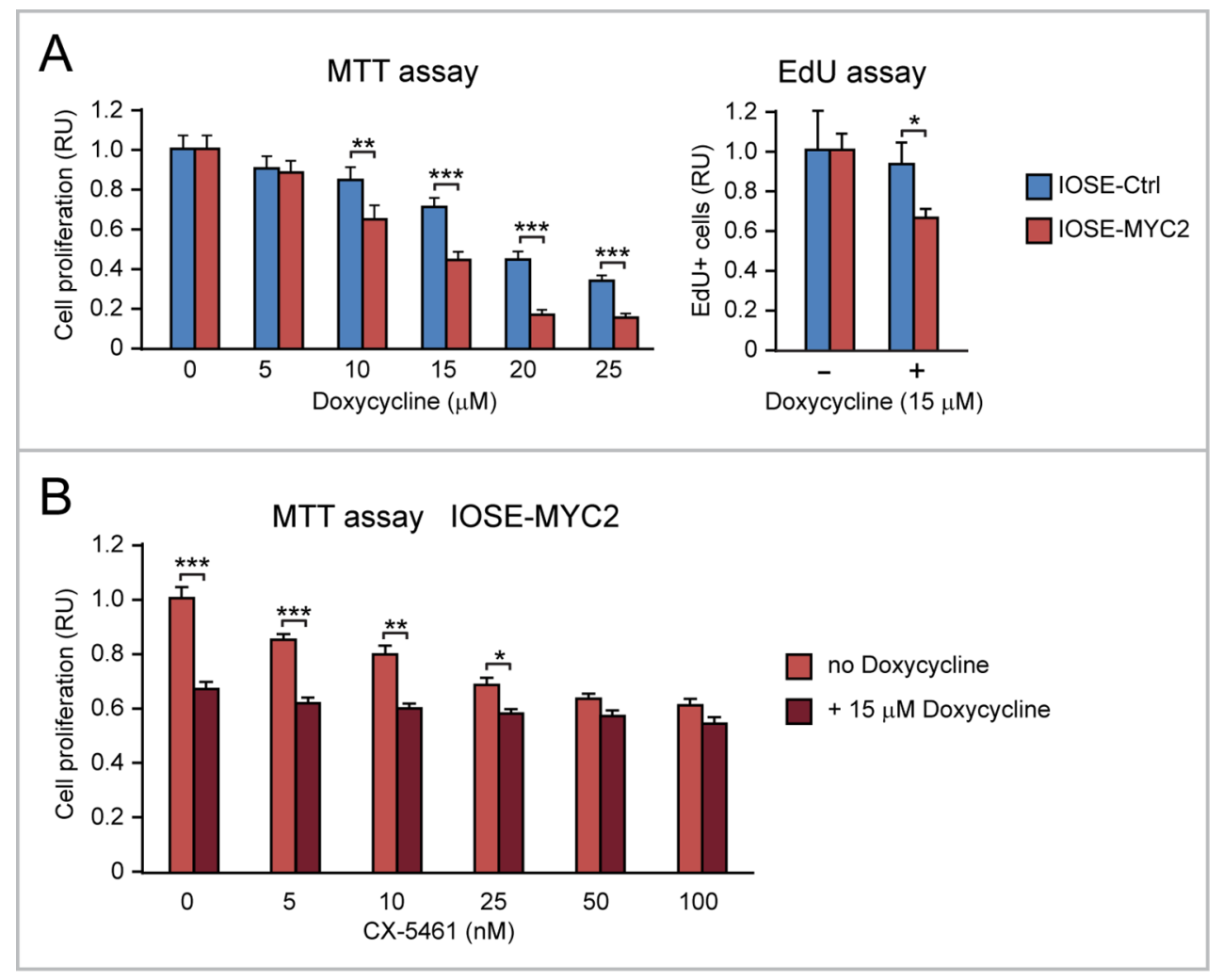

Figure 10: MYC overexpression sensitizes human ovarian epithelial cells to the anti-proliferative action of doxycycline, alone or in combination with CX-5461. (A) Doxycycline inhibits cell proliferation (assessed both by MTT assay, shown on the left, and EdU incorporation assay, shown on the right) significantly more in IOSE-MYC2 than in IOSE-Ctrl. (B) MTT assay showing that doxycycline $(15 \mu \mathrm{M})$ significantly enhances the antiproliferative effect of CX-5461 $(5-25 \mathrm{nM})$ in IOSE-MYC2 cells. ${ }^{*} \mathrm{p}<0.05,{ }^{* * *} \mathrm{p}<0.01$, ${ }^{* * *} \mathrm{p}<0.001$. 
34, 35]. In particular, one of these molecules, CX-5461, which targets Pol I-mediated rRNA transcription, proved to be effective in in vitro and preclinical MYC-driven cancer cell models [16, 21, 22]. CX-5461 is currently tested in clinical trials for advanced hematological malignancies (Australian New Zealand Clinical Trials Registry 12613001061729) and solid tumors (ClinicalTrials.gov Identifier NCT02719977), and its use could be extended to clinical trials of MYC-driven solid tumors, including breast, ovarian and prostate cancer [19, 24, 32].

Interestingly, CX-5461 was shown to be even more effective when used in combination with other drugs, such as drugs targeting PI3K-AKT-mTORC1dependent nucleolar ribosome biogenesis and translation [21]. By the same tenet, we reasoned that CX-5461 could also be more effective if combined with drugs targeting mitochondrial ribogenesis processes and translation in MYC-overexpressing cells. Indeed, recent studies highlighted the anticancer potential of several antiviral and antibacterial molecules capable of targeting either POLRMT-mediated mitochondrial rRNA transcription or mitoribosome function $[7,10,26]$. Specifically, there are several ribonucleosides analogues, originally developed for antiviral therapy, which are substrates and inhibitors of human mitochondrial RNA polymerase (POLRMT) [33], and can be repurposed for cancer therapy. In preclinical studies the 2-C-methyladenosine ribonucleoside (2'-C-MeA) was shown to reduce mitochondrial gene expression and increase cell death in acute myelogenous leukemia (AML), while treatment of normal human

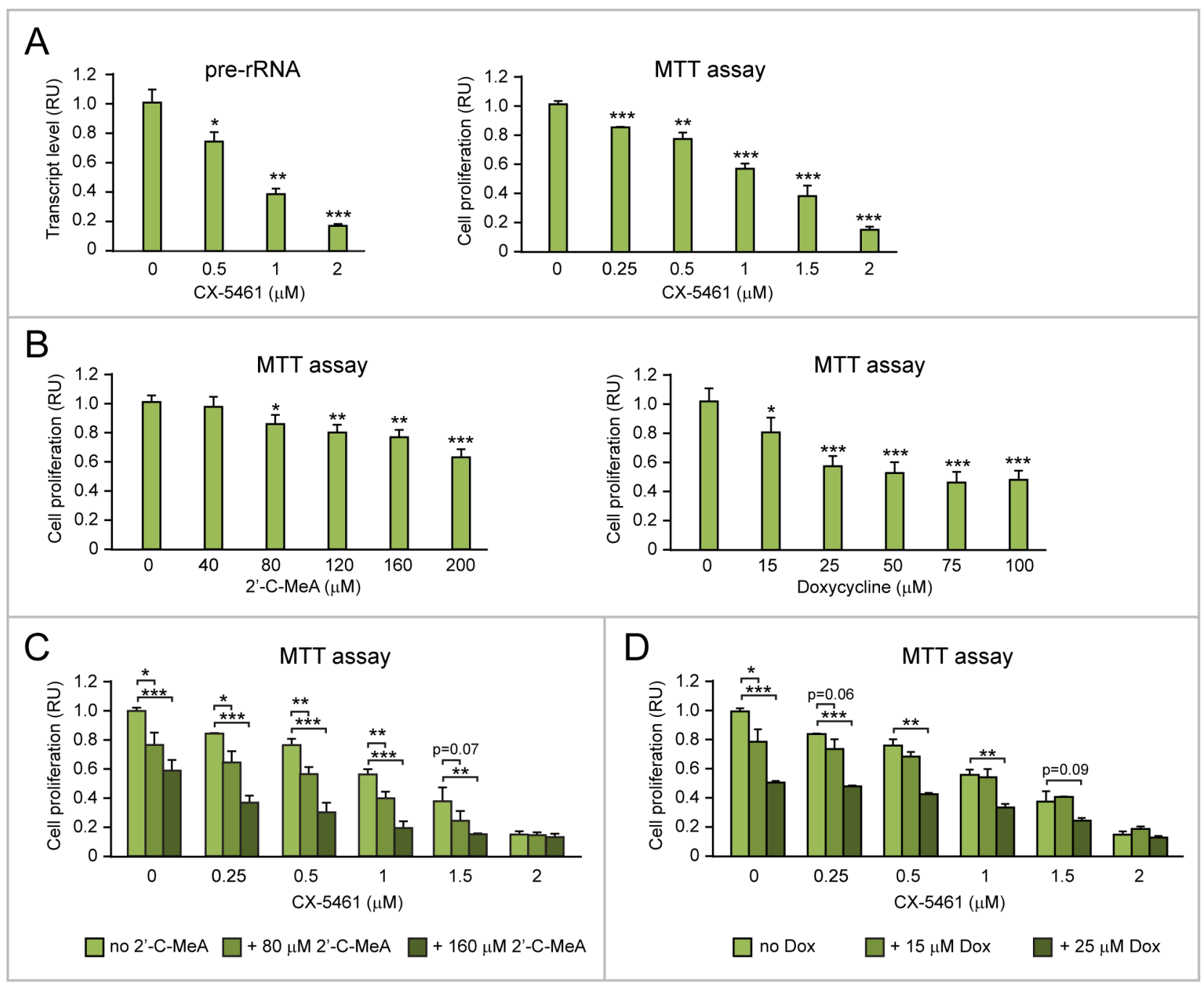

Figure 11: Both 2'-C-MeA and doxycycline can enhance the antiproliferative effects of CX-5461 in MYC-overexpressing ovarian cancer cells. (A) CX-5461, by inhibiting Pol I-mediated rRNA transcription (assessed by pre-rRNA qRT-PCR, left), significantly hinders the proliferation of MYC-overexpressing CAOV4 ovarian cancer cells (assessed by MTT assay, right). (B) Both inhibition of mtrRNA transcription by $2^{\prime} \mathrm{C}-\mathrm{MeA}$ (left) and inhibition of mitoribosome function by doxycycline (right) significantly decrease CAOV4 cell proliferation. (C-D) Both 2'-C-MeA (C) and doxycycline (D) enhance the antiproliferative effects of CX-5461 in CAOV4 cells. " $\mathrm{p}<0.05$, ${ }^{* *} \mathrm{p}<0.01,{ }^{* * *} \mathrm{p}<0.001$. 
hematopoietic cells did not alter clonogenic growth [27]. Due to the ancestral relationship between bacteria and mitochondria, several classes of antibiotics, including tetracyclines (e.g. Doxycycline and Tigecycline) capable of targeting mitoribosome function, are also re-evaluated as potential anti-cancer drugs ([26] and references within).

This study provides the proof of concept that available drugs targeting mitochondrial ribogenesis or mitoribosome function can improve the anticancer effects of emerging new drugs capable of selectively targeting nucleolar Pol I rRNA synthesis in cancer and, in particular, MYC-driven cancer of different histotypes due to concomitant MYC deregulation of both nucleolar and mitochondrial rRNA synthesis and ribogenesis processes.

Based on preliminary observations that ovarian cancer cells overexpressing MYC (CAOV4) displayed upregulation of POLRMT and mt-rRNA synthesis relative to ovarian cancer cells that do not overexpress MYC (CAOV3), we assessed the antiproliferative effects of targeting POLRMT-mediated mt-rRNA transcription in two human ovarian epithelial cell contexts (IOSE and HOSE), and in human fibroblasts (HFF) overexpressing
MYC. Consistent with genetic studies (either MYC or POLRMT knock down), we found that the POLRMT inhibitor 2'-C-MeA significantly reduced both mtrRNA transcription and the number of cells in $\mathrm{S}$ phase. Remarkably, even if MYC-overexpressing ovarian epithelial cells were more sensitive to the anti-proliferative action of the Pol I inhibitor CX-5461 alone, 2'-C-MeA significantly enhanced the effects of CX-5461. Indeed, in the presence of 2'-C-MeA, CX-5461 exerted an antiproliferative effect at lower concentrations (5-10 nM).

Further, based on the fact that MYC upregulation induces PTCD3, MRPS5, and MRPS27 proteins necessary for mitoribogenesis in MYC-overexpressing CAOV4 cancer cells, as well as in HFF fibroblast and different ovarian epithelial cell contexts with MYC overexpression, we tested if doxycycline, a tetracycline that targets the $28 \mathrm{~S}$ subunit of mitochondrial ribosomes, was capable of reducing $\mathrm{MYC}$-induced proliferation. Indeed, we found that MYC-overexpressing fibroblasts and ovarian epithelial cells were more sensitive than control cells to the anti-proliferative action of doxycycline alone. Moreover, doxycycline, when used in combination with

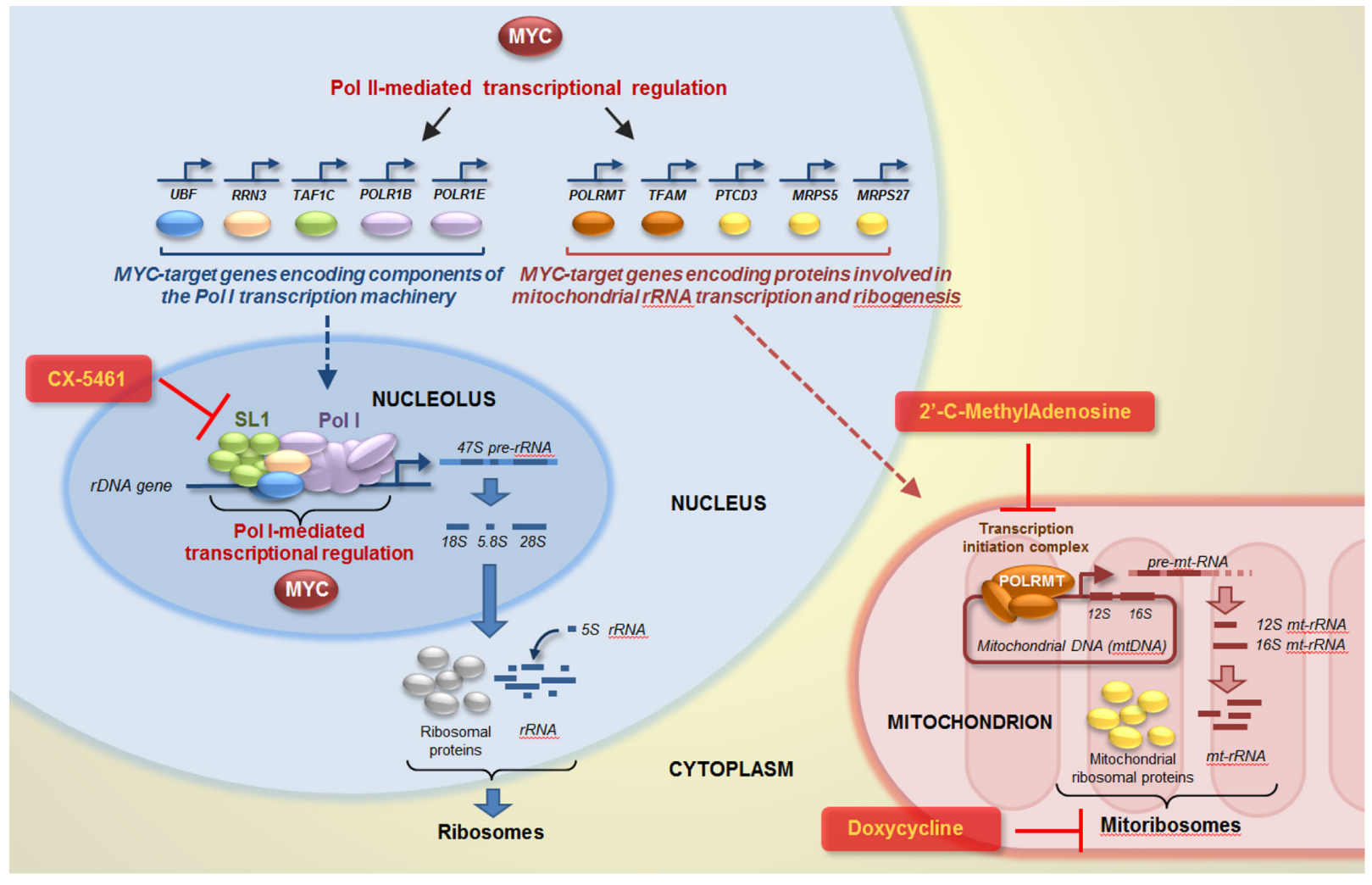

Figure 12: Targeting nucleolar and mitochondrial rRNA transcription and ribogenesis to curb MYC-induced cancer cell proliferation. MYC can promote nucleolar rRNA transcription by directly activating Pol I-mediated transcription of ribosomal genes, as well as by upregulating Pol II-mediated transcription of "Pol I regulon" genes. Concomitantly, MYC can also promote mitoribosome synthesis by upregulating Pol II-mediated transcription of genes encoding factors necessary for mitochondrial rRNA transcription (POLRMT and TFAM) and mitochondrial ribosomal proteins (PTCD3, MRPS5, and MRPS27). Drugs inhibiting Pol I (e.g. CX-5461), mitochondrial rRNA transcription (e.g. 2'-C-MeA), or mitoribosome function (e.g. doxycycline) could be used, alone or in combination, to target MYC-overexpressing cancer cells, such as ovarian cancer cells. 
low doses of CX-5461 Pol I inhibitor, enhanced CX-5461 antiproliferative effect in MYC overexpressing ovarian epithelial cells. In the same cell contexts, we also tested Tigecycline, another tetracycline that was recently shown to curb proliferation of MYC-overexpressing lymphoma and osteosarcoma cells $[7,10]$, but we did not achieve the anti-proliferative effects of doxycycline (data not shown).

Finally, we found that CX-5461 significantly inhibits CAOV4 ovarian cancer cell proliferation, and that the anti-proliferative effect of CX-5461 is significantly enhanced by co-treatment with either 2'C-MeA or doxycycline. Since CX-5461 was recently shown to have a positive effect on both chemoresistant ovarian epithelial cells and patient derived xenografts [36], it is possible that drugs targeting mt-rRNA and mitoribogenesis, when combined with drugs inhibiting Pol I like CX-5461, can be also effective in chemoresistant MYC-overexpressing cancer cell populations.

Overall these findings indicate that leveraging a) MYC-regulated rRNA transcription by RNA polymerases in distinct cell sites (nucleolar Pol I and mitochondrial POLRMT) and b) other MYC-regulated processes and functions of ribogenesis and mitoribogenesis, can be an offbeat approach to control MYC-driven cancers.

\section{MATERIALS AND METHODS}

\section{Cells and cell culture}

HFF-Ctrl (originally named HFF-pB) and HFFMYC human foreskin fibroblast cell lines, kindly obtained from Dr. C. Grandori, Fred Hutchinson Cancer Research Center, Seattle, and previously described in $[3,28]$, were grown in DMEM plus $10 \%$ FBS (Thermo Fisher). CAOV3 and CAOV4 ovarian cancer cells (both from ATCC, Manassas, VA) were grown in DMEM plus 10\% FBS and MCDB105/Media199 1:1 plus 15\% FBS, respectively. Telomerase-immortalized human ovarian surface epithelial cells (IOSE, clone C21), kindly provided by Dr. F. Balkwill, Barts Cancer Institute, Queen Mary University of London, London, were grown in MCDB105/ Media199 1:1 plus 15\% FBS supplemented with $5 \mu \mathrm{g} / \mathrm{ml}$ insulin (Sigma), $10 \mathrm{ng} / \mathrm{ml}$ human EGF (Peprotech), 0.5 $\mu \mathrm{g} / \mathrm{ml}$ hydrocortisone (Sigma), and $34 \mu \mathrm{g}$ protein $/ \mathrm{ml}$ BPE (Lonza) [31]. T1074 SV40-immortalized human ovarian epithelial cells (in this study referred to as HOSE) were purchased from ABM (Richmond, BC, Canada) and grown in MCDB105/Media199 1:1 plus 15\% FBS. All cell lines were grown in a humidified incubator with $5 \%$ $\mathrm{CO}_{2}$ at $37^{\circ} \mathrm{C}$.

IOSE cells stably overexpressing different levels of ectopic MYC (IOSE-MYC and IOSE-MYC2) were developed by lentiviral infection. First, human MYC cDNA was subcloned from pCDH-puro-MYC (Addgene) into the $\mathrm{BamH} \mathrm{I} / \mathrm{Xba}$ I restriction sites of $\mathrm{pCDH}-\mathrm{Hygro}$ (CD515B-1) (System Biosciences). Second, pCDHHygro-MYC was co-transfected along with VSV-G and DeltaR plasmids (both provided by Dr. K. Gurova, Roswell Park Cancer Institute, Buffalo, NY) into HEK293 cells by using lipofectamine LTR (Thermo Fisher) in order to produce lentiviral particles. Medium containing lentiviral particles was harvested after $48 \mathrm{~h}$ and $72 \mathrm{~h}$ and filtered through a $0.45 \mu$ filter. Lentiviral titer was assessed by using Lenti-X Go Stix (Clontech). Finally, $40-50 \%$ confluent IOSE cells were infected with medium containing lentiviral particles diluted 1:2 (IOSE-MYC2) or 1:3 (IOSE-MYC) with growth medium, in the presence of polybrene at a final concentration of $8 \mu \mathrm{g} / \mathrm{ml}$. The infection was repeated after $24 \mathrm{~h}$. After two rounds of infection, cells were trypsinized, seeded in flasks and selected with 0.2 $\mathrm{mg} / \mathrm{ml}$ hygromycin. In parallel, IOSE were infected with empty pCDH-Hygro to obtain IOSE-Ctrl cells. HOSE stably overexpressing ectopic MYC (HOSE-MYC2) were developed by lentiviral infection as described above using pCDH-puro-MYC for lentiviral production and using puromycin $(0.5 \mu \mathrm{g} / \mathrm{ml})$ for selection. Control HOSE were developed by infection with empty pCDH-puro. HFF-pB and HFF-MYC were authenticated by PCR detection of pBABE and pBABE-MYC, respectively. IOSE and HOSE were fingerprinted by STR analysis.

\section{Drugs and treatments}

CX-5461 (ApexBio) was dissolved in $50 \mathrm{mM}$ $\mathrm{NaH}_{2} \mathrm{PO}_{4}(\mathrm{pH} 4.5)$ to obtain a $1 \mathrm{mM}$ stock solution. 2'-C-Methyladenosine (Santa Cruz Biotechnologies) was dissolved in DMSO to obtain a $100 \mathrm{mM}$ stock solution. Doxycycline (Fisher Scientific) was dissolved in $\mathrm{H}_{2} \mathrm{O}$ to obtain a $100 \mathrm{mM}$ stock solution. Drugs were stored in aliquots at $-20^{\circ} \mathrm{C}$. Treatments were performed with drugs or vehicle diluted in medium at the indicated concentrations as described in the Results. For qRT-PCR analysis, EU incorporation, and EdU incorporation, 30$50 \%$ confluent cells were treated for $24 \mathrm{~h}$. For MTT analysis, cells were treated for 3-5 days refreshing the medium every 48 hours for the duration of the treatment.

\section{SiRNA}

$40-60 \%$ confluent cells seeded on 24-well plates were transiently transfected with $10 \mathrm{nM}$ Silencer Select pre-designed siRNAs (Thermo Fisher) targeting MYC (s9129+s9131), UBTF (s14613+s14615), RRN3 (s29324) or POLRMT (s10825+s10826) by using RNAi max Lipofectamine (Thermo Fisher) according to the manufacturer's instructions. As a negative control, cells were transfected with $10 \mathrm{nM}$ siRNA Negative Control (Thermo Fisher). 24-48h after transfection, cells were analyzed by qRT-PCR or EdU incorporation.

\section{Western blot}

Cells were lysed in RIPA Buffer (Cell Signaling) supplemented with $1 \mathrm{mM}$ PMSF and EDTA-free Protease 
Inhibitor Cocktail (Roche). Total protein concentration was measured by using Coomassie Plus Protein Assay Reagent (Thermo Fisher). Equal amounts of proteins were separated by SDS PAGE electrophoresis and transferred onto a PVDF membrane according to standard procedures. Membranes were blocked with 5\% non-fat dry milk diluted in TBS $+0.05 \%$ Tween 20 for $1 \mathrm{~h}$ at room temperature, incubated over night at $4{ }^{\circ} \mathrm{C}$ with anti-MYC (C33) antibody (Santa Cruz Biotechnology) diluted in 5\% milk, washed, incubated for $1 \mathrm{~h}$ at room temperature with HRP-conjugated anti-mouse antibody (GE Healthcare) diluted in 5\% milk, washed, and incubated with Clarity Western ECL Substrate (Bio-Rad). Chemiluminescence was detected and quantified by using ChemiDoc Touch Imaging System (Bio-Rad).

\section{qRT-PCR}

RNA was extracted with Trizol (Thermo Fisher), treated with DNase I (Thermo Fisher), and retrotranscribed by using High Capacity cDNA reverse transcription kit (Thermo Fisher). cDNA was analyzed by real time PCR by using the iQ SYBR Green Supermix (Bio-Rad) and primers amplifying MYC (sense: 5'-cctctcaacgacagcagct-3', antisense: $\quad 5^{\prime}$-cagaaggtgatccagactctg-3'), pre-rRNA 5'ETS (sense: 5'-CTGAGGGAGCTCGTCGGTGT-3', antisense: 5'-GCAGAGCGCCTCCGAAGTCA-3'), UBF (sense: 5'-ACCAAGCCACCTCCGAACAG-3', antisense: 5'-AGGCAGGCTCTCGAGGAAAC-3'), RRN3 (sense: 5'-CGGAAACCTGAAAGAAGGTTTGC-3', antisense: 5'-CTGGCGATTGTTCCTCTCAATG-3'), PTCD3(5'-ACAACAGACTCCATGCTGATGT-3', antisense: 5'-AAGCGAGGGTTCTATTCCAATG-3'), MRPS5 (sense: 5'-TGCCACAGGGCCATCATCAC-3', antisense: 5'-CATGGAGGCCCTTCTTATCAG-3'), MRPS27 (sense: 5'-GGCTATGCACTTCTTGGGAAG-3', antisense: 5'-GC ACATCGAGCGCTTCTCTAC-3'), TAF1C (5'-GGACA GGCTGCATTTCCAAGAG-3', antisense: 5'-AGGTGA GGGCTGAGGCTGAT-3'), POLR1B (5'-TATGGAAG ATGCCATGATTGTGA-3', antisense: 5'-TGTAATACG GATCTCCGTACTG-3'), POLR1E (sense: 5'-GGTGT GACTGCTCTGGTCAG-3', antisense: 5'-GGTGCAAT GGCTGTTCTCCTC-3'), POLRMT (sense: 5'-CATCG AAAGGTGTCTGGAACAG-3', antisense: 5'-CACGCC CATCCTTGGCATAC-3'), TFAM (sense: 5'-CATCTG TCTTGGCAAGTTGTCC-3', antisense: 5'-ACTCCGC CCTATAAGCATCTTG-3'), mtRNA 12S (sense: 5'-GTGAGTTCACCCTCTAAATCAC-3', antisense: 5'-GACTTGGGTTAATCGTGTGACC-3'), mtRNA 16S (sense: 5'-CCTGGTGATAGCTGGTTGTCC-3', antisense: 5'-CAATTGGGTGTGAGGAGTTCAG-3'), mtRNA 1216S (sense: 5'-GAGGAGACAAGTCGTAACATGG-3', antisense: 5'-CTATATCTATTGCGCCAGGTTTC-3'), or GAPDH (sense: 5'-GAAGGTGAAGGTCGGAGTC-3', antisense: 5'-GAAGATGGTGATGGGATTTC-3') as we previously described [20]. Statistical significance was calculated by using the Student's t-test.

\section{Assessment of nucleolar EU incorporation}

5-ethynyl uridine (EU) incorporation assay was performed by using Click-iT RNA Alexa Fluor Imaging Kit (Thermo Fisher). Subconfluent cells were incubated with $1 \mathrm{mM}$ EU under standard growth conditions for $2 \mathrm{~h}$, fixed with 4\% paraformaldehyde for $10 \mathrm{~min}$, permeabilized with PBS $+0.5 \%$ Triton X100 for 15 min. and incubated with Click-iT reaction cocktail for 30 min., blocked with PBS+ (PBS plus 1\% BSA, 1\% FBS, $0.05 \%$ Tween 20 ) for $20 \mathrm{~min}$, incubated with anti-B23 (Santa Cruz) diluted in PBS+ for $1 \mathrm{~h}$ at room temperature, washed four times with $\mathrm{PBS}+$, incubated with antirabbit AlexaFluor 546 (Thermo Fisher) diluted in PBS+ for $1 \mathrm{~h}$, washed four times with PBS, counterstained with $330 \mathrm{nM}$ DAPI for $10 \mathrm{~min}$., and mounted with Vectashield (Vector Laboratories). Cells were imaged with a fluorescence microscope using the same exposure time for each fluorescent channel. EU incorporation in the nucleolar compartment (identified by B23 staining) was assessed with Adobe Photoshop by multiplying the EU-positive nucleolar area (number of pixels) by the EU signal intensity (mean) in the B23-positive nucleolar compartment normalized to the EU signal intensity (mean) in the surrounding nucleoplasm. Statistical significance was calculated by Student's t-test.

\section{MTT assay}

$2-5 \times 10^{3}$ cells/well were seeded in 24-well plates in four replicates, let attach overnight, and treated with the indicated drugs diluted in growth medium. After 3-5 days of treatment, cells were incubated for 2 hours under standard growth conditions with $0.5 \mathrm{mg} / \mathrm{ml}$ 3-(4,5-dimethylthiazol-2-yl)-2,5-diphenyltetrazolium bromide (MTT) (Sigma) and lysed with DMSO. Cell lysates were transferred into a 96- well plate, and absorbance at $570 \mathrm{~nm}$ was measured on a microplate spectrophotometer. Statistical significance was calculated by Student's t-test.

\section{EdU incorporation assay}

EdU-incorporation was assessed with Click-iT EdU imaging kit (Thermo Fisher), according to the manufacturer's instructions. Briefly, cells were incubated under standard growth conditions with $10 \mu \mathrm{M}$ EdU for 1h. After EdU incorporation, cells were fixed with $4 \%$ paraformaldehyde for $10 \mathrm{~min}$., incubated with PBS plus $0.5 \%$ Triton X100 for 10 min., washed with PBS plus $3 \%$ BSA, reacted with Click-iT reaction cocktail for 30 min., counterstained with $330 \mathrm{nM}$ DAPI for $10 \mathrm{~min}$, and mounted with Vectashield (Vector Laboratories). Cells were imaged with a fluorescence microscope. DAPIpositive cells and EdU-positive cells were counted in at least 5 random fields. Statistical significance was calculated by using the Student's t-test. 


\section{Author contributions}

SR contributed to formulating the hypothesis, designed and executed the experiments, analyzed the data, and drafted the manuscript. AJW contributed to the execution of the experiments and data analysis. NS conceived the hypothesis, supervised the overall study, and wrote the manuscript.

\section{ACKNOWLEDGMENTS AND FUNDING}

We thank Dr. C. Grandori, Fred Hutchinson Cancer Research Center, Seattle, for providing us with the HFFMYC and HFF-Ctrl cell lines, Dr. F. Balkwill, Barts Cancer Institute, Queen Mary University of London, London, for providing us with the IOSE cell line; Dr. B Amati, European Institute of Oncology, Milan, for useful discussion; Dr. K. Gurova and L. Prendergast, Roswell Park Cancer Institute, for technical advice and reagents for lentiviral particle production. Funding for this study was provided by the NCI R01 CA127614 grant (NS), an RPCI-UPCI Ovarian Cancer Spore DRP award (NS), and the NCI P30 CA016056 institutional grant.

\section{CONFLICTS OF INTEREST}

The authors declare no conflicts of interest.

\section{REFERENCES}

1. Campbell KJ, White RJ. MYC regulation of cell growth through control of transcription by RNA polymerases I and III. Cold Spring Harb Perspect Med. 2014. https://doi. org/10.1101/cshperspect.a018408.

2. Arabi A, Wu S, Ridderstrale K, Bierhoff H, Shiue C, Fatyol K, Fahlen S, Hydbring P, Soderberg O, Grummt I, Larsson LG, Wright AP. c-Myc associates with ribosomal DNA and activates RNA polymerase I transcription. Nat Cell Biol. 2005; 7:303-10.

3. Grandori C, Gomez-Roman N, Felton-Edkins ZA, Ngouenet C, Galloway DA, Eisenman RN, White RJ. c-Myc binds to human ribosomal DNA and stimulates transcription of rRNA genes by RNA polymerase I. Nat Cell Biol. 2005; $7: 311-8$,

4. Grewal SS, Li L, Orian A, Eisenman RN, Edgar BA. Mycdependent regulation of ribosomal RNA synthesis during Drosophila development. Nat Cell Biol. 2005; 7:295-302.

5. Poortinga G, Hannan KM, Snelling H, Walkley CR, Jenkins A, Sharkey K, Wall M, Brandenburger Y, Palatsides M, Pearson RB, McArthur GA, Hannan RD. MAD1 and c-MYC regulate UBF and rDNA transcription during granulocyte differentiation. EMBO J. 2004; 23:3325-35. https://doi.org/10.1038/sj.emboj.7600335.

6. Poortinga G, Wall M, Sanij E, Siwicki K, Ellul J, Brown D, Holloway TP, Hannan RD, McArthur GA. c-MYC coordinately regulates ribosomal gene chromatin remodeling and Pol I availability during granulocyte differentiation. Nucleic Acids Res. 2011; 39:3267-81. https://doi.org/10.1093/nar/gkq1205.

7. Oran AR, Adams CM, Zhang XY, Gennaro VJ, Pfeiffer HK, Mellert HS, Seidel HE, Mascioli K, Kaplan J, Gaballa MR, Shen C, Rigoutsos I, King MP, et al. Multi-focal control of mitochondrial gene expression by oncogenic MYC provides potential therapeutic targets in cancer. Oncotarget. 2016; 7:72395-414. https://doi.org/10.18632/oncotarget.11718.

8. Li F, Wang Y, Zeller KI, Potter JJ, Wonsey DR, O'Donnell KA, Kim JW, Yustein JT, Lee LA, Dang CV. Myc stimulates nuclearly encoded mitochondrial genes and mitochondrial biogenesis. Mol Cell Biol. 2005; 25:6225-34. https://doi. org/10.1128/MCB.25.14.6225-6234.2005.

9. Bestwick ML, Shadel GS. Accessorizing the human mitochondrial transcription machinery. Trends Biochem Sci. 2013; 38:283-91. https://doi.org/10.1016/j.tibs.2013.03.006.

10. D'Andrea A, Gritti I, Nicoli P, Giorgio M, Doni M, Conti A, Bianchi V, Casoli L, Sabo A, Mironov A, Beznoussenko $\mathrm{GV}$, Amati B. The mitochondrial translation machinery as a therapeutic target in Myc-driven lymphomas. Oncotarget. 2016; 7:72415-30. https://doi.org/10.18632/ oncotarget.11719.

11. Dang CV. MYC on the path to cancer. Cell. 2012; 149:2235. https://doi.org/10.1016/j.cell.2012.03.003.

12. Gabay M, Li Y, Felsher DW. MYC activation is a hallmark of cancer initiation and maintenance. Cold Spring Harb Perspect Med. 2014. https://doi.org/10.1101/cshperspect. a014241.

13. Poortinga G, Quinn LM, Hannan RD. Targeting RNA polymerase I to treat MYC-driven cancer. Oncogene. 2015; 34:403-12. https://doi.org/10.1038/onc.2014.13.

14. Ruggero D. Revisiting the nucleolus: from marker to dynamic integrator of cancer signaling. Sci Signal. 2012; 5:pe38.

15. Bywater MJ, Pearson RB, McArthur GA, Hannan RD. Dysregulation of the basal RNA polymerase transcription apparatus in cancer. Nat Rev Cancer. 2013; 13:299-314. https://doi.org/10.1038/nrc3496.

16. Bywater MJ, Poortinga G, Sanij E, Hein N, Peck A, Cullinane C, Wall M, Cluse L, Drygin D, Anderes K, Huser N, Proffitt C, Bliesath J, et al. Inhibition of RNA polymerase I as a therapeutic strategy to promote cancerspecific activation of p53. Cancer Cell. 2012; 22:51-65.

17. Drygin D, Lin A, Bliesath J, Ho CB, O'Brien SE, Proffitt C, Omori M, Haddach M, Schwaebe MK, Siddiqui-Jain A, Streiner N, Quin JE, Sanij E, et al. Targeting RNA polymerase I with an oral small molecule CX-5461 inhibits ribosomal RNA synthesis and solid tumor growth. Cancer Res. 2011; 71:1418-30.

18. Hein N, Cameron DP, Hannan KM, Nguyen NN, Fong CY, Sornkom J, Wall M, Pavy M, Cullinane C, Diesch J, Devlin JR, George AJ, Sanij E, et al. Inhibition of Pol I 
transcription treats murine and human AML by targeting the leukemia-initiating cell population. Blood. 2017; 129:288295. https://doi.org/10.1182/blood-2016-05-718171.

19. Quin JE, Devlin JR, Cameron D, Hannan KM, Pearson RB, Hannan RD. Targeting the nucleolus for cancer intervention. Biochim Biophys Acta. 2014; 1842:802-16. https://doi. org/10.1016/j.bbadis.2013.12.009.

20. Rossetti S, Wierzbicki AJ, Sacchi N. Mammary epithelial morphogenesis and early breast cancer. Evidence of involvement of basal components of the RNA Polymerase I transcription machinery. Cell Cycle. 2016; 15:2515-26. https://doi.org/10.1080/15384101.2016.1215385.

21. Devlin JR, Hannan KM, Hein N, Cullinane C, Kusnadi E, Ng PY, George AJ, Shortt J, Bywater MJ, Poortinga G, Sanij E, Kang J, Drygin D, et al. Combination therapy targeting ribosome biogenesis and mRNA translation synergistically extends survival in MYC-driven lymphoma. Cancer Discov. 2016; 6:59-70. https://doi.org/10.1158/2159-8290. CD-14-0673.

22. Lee HC, Wang H, Baladandayuthapani V, Lin H, He J, Jones RJ, Kuiatse I, Gu D, Wang Z, Ma W, Lim J, O'Brien S, Keats J, et al. RNA polymerase I inhibition with CX-5461 as a novel therapeutic strategy to target MYC in multiple myeloma. Br J Haematol. 2017; 177:80-94. https:/doi. org/10.1111/bjh.14525.

23. Rebello RJ, Kusnadi E, Cameron DP, Pearson HB, Lesmana A, Devlin JR, Drygin D, Clark AK, Porter L, Pedersen J, Sandhu S, Risbridger GP, Pearson RB, et al. The dual inhibition of RNA pol I transcription and PIM kinase as a new therapeutic approach to treat advanced prostate cancer. Clin Cancer Res. 2016; 22:5539-52. https://doi. org/10.1158/1078-0432.CCR-16-0124.

24. Rebello RJ, Pearson RB, Hannan RD, Furic L. Therapeutic approaches targeting MYC-driven prostate cancer. Genes (Basel). 2017. https://doi.org/10.3390/genes8020071.

25. Kim HJ, Maiti P, Barrientos A. Mitochondrial ribosomes in cancer. Semin Cancer Biol. 2017. https://doi.org/10.1016/j. semcancer.2017.04.004.

26. Lamb R, Ozsvari B, Lisanti CL, Tanowitz HB, Howell A, Martinez-Outschoorn UE, Sotgia F, Lisanti MP. Antibiotics that target mitochondria effectively eradicate cancer stem cells, across multiple tumor types: treating cancer like an infectious disease. Oncotarget. 2015; 6:4569-84. https://doi. org/10.18632/oncotarget.3174.

27. Bralha FN, Liyanage SU, Hurren R, Wang X, Son MH, Fung TA, Chingcuanco FB, Tung AY, Andreazza AC,
Psarianos P, Schimmer AD, Salmena L, Laposa RR. Targeting mitochondrial RNA polymerase in acute myeloid leukemia. Oncotarget. 2015; 6:37216-28. https://doi. org/10.18632/oncotarget.6129.

28. Benanti JA, Wang ML, Myers HE, Robinson KL, Grandori C, Galloway DA. Epigenetic down-regulation of ARF expression is a selection step in immortalization of human fibroblasts by c-Myc. Mol Cancer Res. 2007; 5:1181-9.

29. Rossetti S, Hoogeveen AT, Esposito J, Sacchi N. Loss of MTG16a (CBFA2T3), a novel rDNA repressor, leads to increased ribogenesis and disruption of breast acinar morphogenesis. J Cell Mol Med. 2010; 14:1358-70.

30. Kalkat M, De Melo J, Hickman KA, Lourenco C, Redel C, Resetca D, Tamachi A, Tu WB, Penn LZ. MYC deregulation in primary human cancers. Genes (Basel). 2017; 8. https://doi.org/10.3390/genes8060151.

31. Li NF, Broad S, Lu YJ, Yang JS, Watson R, Hagemann T, Wilbanks G, Jacobs I, Balkwill F, Dafou D, Gayther SA. Human ovarian surface epithelial cells immortalized with hTERT maintain functional $\mathrm{pRb}$ and $\mathrm{p} 53$ expression. Cell Prolif. 2007; 40:780-94. https://doi. org/10.1111/j.1365-2184.2007.00462.x.

32. Yan S, Frank D, Son J, Hannan KM, Hannan RD, Chan KT, Pearson RB, Sanij E. The potential of targeting ribosome biogenesis in high-grade serous ovarian cancer. Int J Mol Sci. 2017. https://doi.org/10.3390/ijms18010210.

33. Arnold JJ, Sharma SD, Feng JY, Ray AS, Smidansky ED, Kireeva ML, Cho A, Perry J, Vela JE, Park Y, Xu Y, Tian Y, Babusis D, et al. Sensitivity of mitochondrial transcription and resistance of RNA polymerase II dependent nuclear transcription to antiviral ribonucleosides. PLoS Pathog. 2012; 8:e1003030. https://doi.org/10.1371/journal. ppat. 1003030 .

34. Hein N, Hannan KM, George AJ, Sanij E, Hannan RD. The nucleolus: an emerging target for cancer therapy. Trends Mol Med. 2013.

35. Hannan RD, Drygin D, Pearson RB. Targeting RNA polymerase I transcription and the nucleolus for cancer therapy. Expert Opin Ther Targets. 2013; 17:873-8.

36. Cornelison R, Dobbin ZC, Katre AA, Jeong DH, Zhang Y, Chen D, Petrova Y, Llaneza DC, Steg AD, Parsons L, Schneider D, Landen CN. Targeting Rna-polymerase I in both chemosensitive and chemoresistant populations in epithelial ovarian cancer. Clin Cancer Res. 2017. https:// doi.org/10.1158/1078-0432.CCR-17-0282. 